Portland State University

PDXScholar

\title{
Reduced Order Description of Experimental Two- Phase Pipe Flows: Characterization of Flow Structures and Dynamics via Proper Orthogonal Decomposition
}

Bianca Fontanin Viggiano

Portland State University

Follow this and additional works at: https://pdxscholar.library.pdx.edu/open_access_etds

Part of the Mechanical Engineering Commons

Let us know how access to this document benefits you.

\section{Recommended Citation}

Viggiano, Bianca Fontanin, "Reduced Order Description of Experimental Two-Phase Pipe Flows: Characterization of Flow Structures and Dynamics via Proper Orthogonal Decomposition" (2017). Dissertations and Theses. Paper 3829.

https://doi.org/10.15760/etd.5723

This Thesis is brought to you for free and open access. It has been accepted for inclusion in Dissertations and Theses by an authorized administrator of PDXScholar. Please contact us if we can make this document more accessible: pdxscholar@pdx.edu. 


\title{
Reduced Order Description of Experimental Two-Phase Pipe Flows: Characterization of Flow Structures and Dynamics via Proper Orthogonal Decomposition
}

$$
\text { by }
$$

\section{Bianca Fontanin Viggiano}

A thesis submitted in partial fulfillment of the requirements for the degree of

\author{
Master of Science \\ in \\ Mechanical Engineering
}

Thesis Committee:

Raúl Bayoán Cal, Chair

Gerald Recktenwald

Derek Tretheway

Portland State University

2017 


\begin{abstract}
Multiphase pipe flow is investigated using proper orthogonal decomposition for tomographic X-ray data, where holdup, cross-sectional phase distributions and phase interface characteristics within the pipe are obtained. Six cases of stratified and mixed flow with water content of $10 \%, 30 \%$ and $80 \%$ are investigated to gain insight into effects of velocity and proportion of water on the flow fields. Dispersed and slug flows are separately analyzed to consider the added interface complexity of the flow fields. These regimes are also highly applicable to industry operational flows. Instantaneous and fluctuating phase fractions of the four flow regime are analyzed and reduced order dynamical descriptions are generated. Stratified flow cases display coherent structures that highlight the liquid-liquid interface location while the mixed flow cases show minimal coherence of the eigenmodes. The dispersed flow displays coherent structures for the first few modes near the horizontal center of the pipe, representing the liquidliquid interface location while the slug flow case shows coherent structures that correspond to the cyclical formation and break up of the slug in the first 5 modes. The low order descriptions of the high water content, stratified flow field indicates that main characteristics can be captured with minimal degrees of freedom. Reconstructions of the dispersed flow and slug flow cases indicate that dominant features are observed in the low order dynamical description utilizing less than $1 \%$ of the full order model. POD temporal coefficients $a_{1}, a_{2}$ and $a_{3}$ show a high level of interdependence for the slug flow case. The coefficients also describe the phase fraction holdup as a function of time for both dispersed and slug flow. The second coefficient, $a_{2}$, and the centerline holdup
\end{abstract}


profile show a mean percent difference below $9 \%$ between the two curves. The mathematical description obtained from the decomposition will deepen the understanding of multiphase flow characteristics and is applicable to long distance multiphase transport pipelines, fluidized beds, hydroelectric power and nuclear processes to name a few. 


\section{Acknowledgements}

I would like to express my sincerest gratitude to my advisors Raúl Bayoán Cal and Murat Tutkun for continuous guidance and support of our research. I would also like to thank members of the research group, including Nicholas Hamilton, Naseem Ali and Elizabeth Camp for new perspectives as well as expertise and insight from their studies. Finally, thank you to the Institute for Energy Technology and specifically Heiner Schümann and Olaf Skjæraasen, for their technical support and guidance from overseas on the multiphase experimental data collection and post-processing. This research could not have been completed without funding from the Hydro Research Foundation and Maseeh College of Engineering at Portland State University. 


\section{Contents}

Abstract

Acknowledgements iii

List of Tables $\quad$ vi

List of Figures vii

$\begin{array}{ll}\text { Nomenclature } & \mathbf{x}\end{array}$

1 Introduction and Motivation 1

$\begin{array}{llr}2 & \text { Theory } & 7\end{array}$

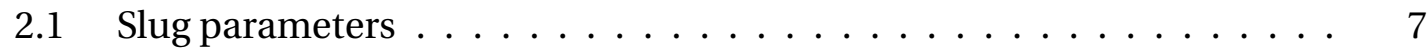

2.2 Proper orthogonal decomposition $\ldots \ldots \ldots \ldots$

3 Experimental Setup $\quad 12$

3.1 Well Flow Loop . . . . . . . . . . . . . . . . . . 12

3.2 X-ray computed tomography system . . . . . . . . . . . . . . . . 13

4 Results: Stratified and Mixed Flow $\quad 15$

4.1 Time-averaged statistics $\ldots \ldots \ldots \ldots \ldots \ldots \ldots \ldots$ 
4.2 Proper orthogonal decomposition . . . . . . . . . . . . . 17

5 Results: Dispersed and Slug Flow 22

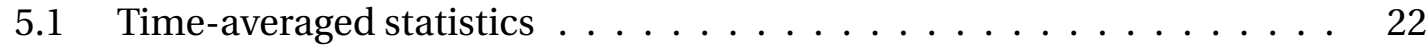

5.2 Proper orthogonal decomposition . . . . . . . . . . . . 24

6 Conclusion $\quad 35$

$\begin{array}{lll}7 & \text { Future Work } & 37\end{array}$

$\begin{array}{ll}\text { Bibliography } & 39\end{array}$ 


\section{List of Tables}

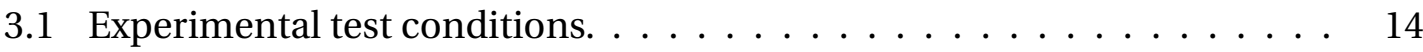

4.1 Corresponding eigenmodes required for $50 \%, 75 \%$ and $95 \%$ reconstruction for the stratified and mixed flow cases. $\ldots \ldots \ldots \ldots \ldots$ 


\section{List of Figures}

1.1 Side view of two-phase flow for visualization of dynamics for various multiphase regimes. .................... 1

2.1 Diagram of the evolution of a liquid slug in pipe. . . . . . . . 7

$3.1 \quad$ Experimental test section (not to scale) . . . . . . . . . . . 12

3.2 Orientation of source and detection devices for one X-ray system along

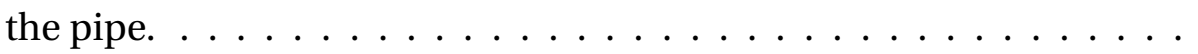

4.1 Time-averaged tomograms of the cross-sectional water distribution for stratified and mixed flow cases. The time-averaged tomograms show the most prominent characteristics of the flow field. Contour lines indicate the local water fraction in steps of $0.1 . \ldots \ldots \ldots$

4.2 The distribution of variance described by (A) normalized eigenvalues, $K_{n}$, and (B) successive summation of eigenvalues, $Z_{n}$ as a function of the mode, $n$. The value associated with each eigenvalues is representative of the amount of information present in the corresponding eigen-

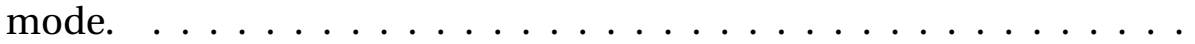


4.3 Eigenmodes 1, 2, 3 and 10 for all cases. The modes show the most dominant structures of the flow with respect to spatial location. The most important structures are present in the first mode. . . . . . . . . . . . . 20

4.4 Phase fraction interactions, $\left\langle\phi_{w}^{\prime} \phi_{o}^{\prime}\right\rangle$, of $05_{\nu} 80_{w c}$ for full order description (left) and the 50\% reconstructed reduced order description (right). The low order description shows the main features of the interaction between the two fluids. The reconstruction uses a single mode while the full order description requires all 30 degrees of freedom of the sys-

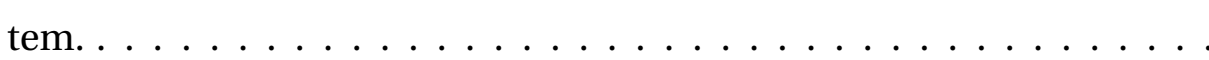

5.1 Mean phase fraction tomograms of the cross-sectional distribution (A) $\left\langle\phi_{w}\right\rangle$ for dispersed flow and (B) $\left\langle\phi_{o}\right\rangle$ for slug flow. Most prominent features of the phase fraction field are depicted. Contour lines indicate the local water fraction in steps of $0.1 \ldots \ldots . \ldots 23$

5.2 The distribution of variance described by (A) normalized eigenvalues, $K_{n}$, and (B) successive summation of eigenvalues, $Z_{n}$ as a function of the mode, $n$. The value associated with each eigenvalues is representative of the amount of important structures present in the correspond-

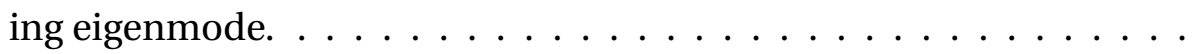

5.3 Eigenmodes 1, 2, 3, 5, 10 and 100 for the dispersed case depicted in (A) through $(\mathrm{F})$, respectively. The modes show the most dominant structures of the flow in the cross-section of the pipe. The kernel of the POD is the instantaneous phase fraction therefore the most prominent structure is the mean phase fraction, shown in the first mode. . . . . 
5.4 Eigenmodes 1, 2, 3, 5, 10 and 100 for the slug flow case depicted in (A) through (F), respectively. The first mode shows the mean phase fraction of the slug flow with successive modes depicting the main features of the flow spatially. . . . . . . . . . . . . . . . . 27

5.5 (A) Full order instantaneous phase fraction tomograms and (B) reduced order instantaneous phase fraction tomograms of dispersed flow, reconstructed with 1 eigenmode. The low order description shows main features of the flow while using less than $1 \%$ of the information from the full order model. . . . . . . . . . . . . .

5.6 (A) Full order instantaneous phase fraction tomograms and (B) reduced order instantaneous phase fraction tomograms of slug flow, reconstructed with 3 eigenmodes. The reduced order dynamical description includes prominent flow features using three degrees of freedom. The full order description of the flow uses 1250 degrees of freedom. . .

5.7 Phase correlation diagrams of temporal coefficients for $i=1,2 \& 3$ for dispersed flow (left column) and slug flow (right column). The asterisk and the square, included in the figures of slug flow, represent the formation at $t=t_{o}$ and break up at $t=t_{f}$ of the slug, respectively. . .

5.8 Experimental holdup, centerline (CL) holdup and $a_{i}$ as a function of time for dispersed and slug flow in (a) and (b), respectively, where $i=$ $1,2,3 \& 4$. The coefficients correspond to the main features of the flow with respect to time. $\ldots \ldots \ldots \ldots \ldots \ldots$ 


\section{Nomenclature}

$\begin{array}{ll}a_{i} & i^{t h} \text { temporal coefficient } \\ D & \text { Diameter of the pipe, } \mathrm{m} \\ \lambda_{i} & i^{t h} \text { eigenvalue } \\ L_{S} & \text { Liquid slug length, } \mathrm{m} \\ v_{S} & \text { Frequency of slug, } \mathrm{s}^{-1} \\ \phi_{w} & \text { Phase fraction of water by volume } \\ \phi_{o} & \text { Phase fraction of oil by volume } \\ \Psi^{i} & i^{t h} \text { eigenmode } \\ t & \text { Time, } \mathrm{s} \\ U_{o} & \text { Velocity of oil phase, } \mathrm{m} / \mathrm{s} \\ U_{g} & \text { Velocity of gas phase, } \mathrm{m} / \mathrm{s}\end{array}$




\section{Chapter 1}

\section{Introduction and Motivation}

Multiphase flows appear in a variety of industrial applications including; petroleum production and transportation systems, production of polymers and other materials, fluidized beds, boiler and heat exchangers tubes and hydroelectric power $[3,6,9,15$, 19,21]. Multiphase flow has complex characteristics such as differences in pressure and density at the interface that hinder numerical simulation accuracy. Stratified flow, shown in Figure 1.1(A), is considered to be the least complex multiphase flow regime though the interface may become chaotic and complex.

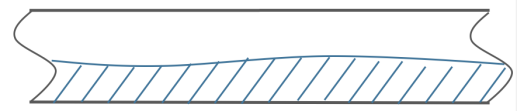

(A) Stratified flow

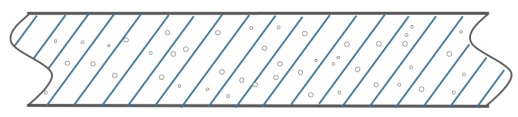

(C) Mixed flow

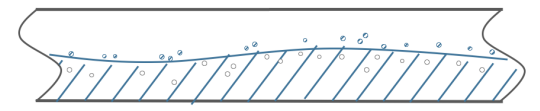

(B) Dispersed flow

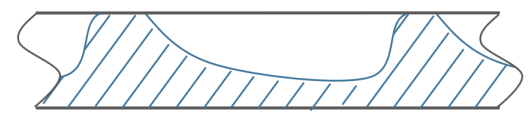

(D) Slug flow

Figure 1.1: Side view of two-phase flow for visualization of dynamics for various multiphase regimes.

The understanding of interface characteristics is limited, primarily due to the lack of analysis performed on experimental stratified flow [15]. Simulators are based on sim- 
plified models or assumptions concerning the interfacial and dispersion behavior of the phenomena, both of which hinder accuracy of the predictions.

Dispersed flow, shown in Figure 1.1(B), is characterized by the distribution of one fluid, the dispersed phase, within another fluid, the carrier phase. Although interface development is considered of secondary importance to the particle size spectra in most dispersed flow analysis, there is correlation between the geometric characteristics of the interface and droplet break up and coalescence [3]. Dispersed flow is often analyzed via numerical simulations but the complexity and scale of the dispersed fluid deem these approaches either inaccurate at the macroscale level or challenging and computationally expensive at the fully resolved level [3]. Mixed flow occurs when there is not a well defined interface between the two fluids, as seen in Figure 1.1(C).

Phase inversion is a phenomenon where the dispersed phase and the carrier phase spontaneously invert due to a small change in the operational conditions. Brauner and Ulmann [4] formulated a model for phase inversion in two-phase pipe flows. The model provides explanations of features of the phonemena in liquid-liquid pipe flows and static mixers and compares favorably with available data on critical holdup for phase inversion. Liu et al. [22] used laser-induced fluorescence to study flow structures in liquid-liquid flows at high dispersed phase fractions. An unstable range was found in which oil-in-water and water-in-oil dispersions could co-exist. This unstable range was different from the ambivalent range seen in agitated flow systems, despite the similar appearance.

Slug flow is intermittent two- or three-phase flow and exists for a wide range of flow rates. A two fluid representation of the flow field is depicted in Figure 1.1(D). Slug flow originates from stratified two-phase flow where small perturbations create 
interfacial waves to grow via Kelvin-Helmoltz instabilities. This causes one phase to entirely occupy the cross-section of the pipe [27]. Other causes of slug flow include accumulation of liquid at nonuniform terrain valleys [1] as well as wave coalescence at high flow rates [28].

In past studies of slug flow, characterization of the flow was carried out via slug length and frequency, gas and liquid holdups and velocity fluctuations. Liquid holdup refers to the percent liquid at an area of interest in the flow. The parameters are analyzed with the intent of tracking and predicting slug development. Understanding the formation and break up of slugs is critical in mitigating the intermittent loading it inflicts on pipeline infrastructure. Serious problems occur as a result of pipe wall damages, especially in hydrocarbon production and transportation lines, leading to severe safety risks [20].

Numerical and experimental studies have been performed to improve and adapt current slug length and shape distribution predictions within the flow field $[6,8,27]$. The two-fluid model, used frequently in numerical analysis, is formulated by considering the cross-sectional averaged governing equations of mass and momentum for each phase. Issa and Kempf [17] took a mechanistic approach to the prediction of slug development, based on the numerical solution of the one-dimensional transient two-fluid model equations. This approach minimized the need for phenomenological models. Given the simplicity of the model, the results of the computations for slug characteristics and data obtained from literature have remarkable agreement.

A one-dimensional two-fluid model was also used in a study by Hanyang and Liejin [12] to investigate a viscous Kelvin-Helmholtz criterion of interfacial wave instability. This study utilized a more complex closure relation including dynamic pressure terms 
in lieu of hydrostatic approximation. The criterion predicts the stability limit of the flow well in horizontal and nearly horizontal pipes. Predicted and experimental results also show that critical liquid height is insensitive to small pipe inclinations, but at low gas velocities, critical liquid velocity and wave velocity are sensitive to small pipe inclinations.

Carneiro et al. [6] examined slug flow numerically and experimentally. The slug flow was simulated with a two-fluid model and verified via experimental measurements. The two-fluid model was used to predict frequency, velocity and slug length which showed good agreement to the experimental measurements with differences varying from $10 \%$ to $20 \%$ for frequency and slug length. Hu et al. [15] used a fastresponse X-ray tomography system to analyze the flow structure and phase distribution in two- and three-phase experimental stratified and slug flows. The experimental results suggested that the commonly used one-dimensional two-fluid model should not only account for cross-sectional distribution of phase fraction and velocity, but also their axial variation.

Hydroelectric power technology, an industrial application of multiphase flow, has proved to be a highly flexible and controllable means of power production. Though the technology is mature, there exists several fundamental fluid flow problems which prevent running of a more cost effective plant. The widely used Francis turbine experiences significant drops in efficiency when operating in off-design conditions [13]. Escalera et al. [10] experimentally investigated hydraulic turbines to evaluate the detection of cavitation. It was found that the bubble growth could be approximated by the generalized Rayleigh-Plesset equation. The use of this equation requires that the bubble pressure and infinite domain pressure are known. Bajic [2] analytically for- 
mulated a novel technique for vibro-acoustical diagnostics of turbine cavitation and demonstrated its use on a Francis turbine. Diagnostic results formed the basis for setting up a high-sensitivity cavitation monitoring system. Predictive maintenance management systems have been studied by Fu et al. [11], with the introduction of a intelligent-control-maintenance-management system platform. Tests are run on an artificial model with results showing that the proposed strategy can guarantee ideal performance.

Proper orthogonal decomposition (POD) can be used to characterize the complex multiphase flow mechanics to understand the dynamical field. Cizmas et al. [7] studied the interactions of gas and solid phases in fluidized beds to explore the implementation of a reduced order model via Galerkin methods. The data were obtained using the Multiphase Flow with Interface eXchange code to simulate the two-dimensional fluidization flow field. Cizmas et al. found that the most dominant characteristics of motion can be captured by a small number of POD modes. As expected, in order to capture the fine details of the spatial features of the flow, a large number of modes are needed. Phase correlation diagrams revealed the existence of a low-dimensional attractor due to the closed circular behavior of the coefficients $a_{2}$ and $a_{3}$ with respect to $a_{1}$.

Brenner et al. [5] studied isothermal and non-isothermal multiphase flow in fluidized beds. Brenner et al. present the derivation and implementation of a reducedorder model (ROM) based on proper orthogonal decomposition. Two methods are utilized for clustering snapshots in the transient region, a coupled and split approach. The split method required an autocorrelation matrix to be computed for each variable. In the coupled method, prior to computation the variables are concatenated. 
The study found the split approach to yield less error for the given case.

The POD examples discussed in the previous paragraph use simulation data to construct the kernel of POD formulation and subsequent analysis. This study implements proper orthogonal decomposition on measurement data from stratified, mixed, dispersed and slug flow in a large diameter pipe. By using experimental multiphase flow data, the mathematical representation pertains to parameters of the flow field. More specifically, all characteristics observed through this analysis are directly related to the flow dynamics, no assumptions are hindering the accuracy of the resulting mathematical descriptions.

Four flow regimes are investigated. For stratified and mixed flow, cases with a water content percentage of $10 \%, 30 \%$ and $80 \%$ are considered, six cases in total. These cases are analyzed to identify the features of the flow that vary as a function of the velocity of the fluid as well as the water-to-oil ratio in the pipe. Dispersed and slug flow are then investigated to gain insight into dynamics of more complex flow fields. These regimes are also prevalent in industrial applications. The characterization of these regimes can lead to increased efficiency of transportation systems of petroleum, tube and shell heat exchangers and polymer production systems to name a few. In particular, the identification of slug initiation and evolution can be used mitigate the irregular loading on pipe infrastructure. 


\section{Chapter 2}

\section{Theory}

\subsection{Slug parameters}

Figure 2.1 depicts the initiation and evolution of a liquid slug and subsequent bubble formation. The gas bubbles are shown in white and form along the top of the pipe. The liquid phase is described by the hatching in the pipe. The schematic includes the pipe diameter, $D$, the length of the liquid slug, $L_{S}$, and the length of the total evolution of the slug, $L$, as well as the gas and liquid velocities, $U_{g}$ and $U_{l}$, respectively. Two liquid slugs are shown by the liquid height reaching the top of the pipe. Another important parameter, the phase fraction, $\phi$, describes the amount of the phase at a given location and time as a percentage from 0 to 1 . Liquid holdup is synonymous with phase fraction, but specific to the liquid phase.

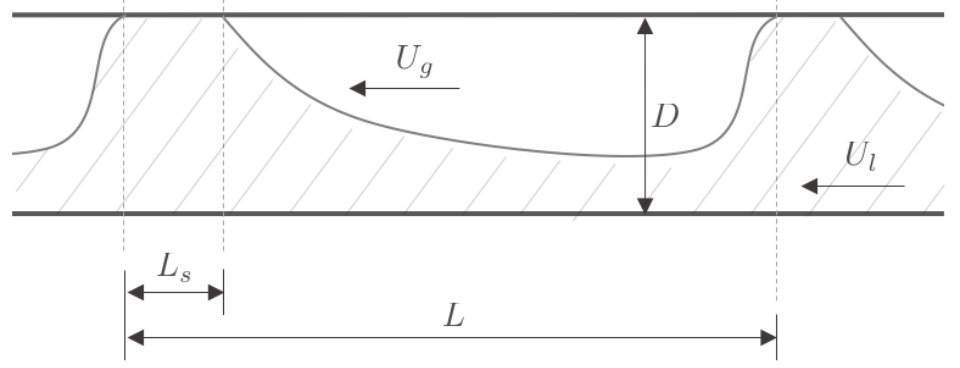

Figure 2.1: Diagram of the evolution of a liquid slug in pipe. 
Given the velocity of the liquid phase, and considering it to be constant throughout the pipe, the length of slug $k$ is

$$
L_{S, k}=U_{l} \Delta t_{a \rightarrow b},
$$

where $U_{l}$ is the liquid velocity and time, $t$, is taken from the head, $a$, to the tail, $b$, of the slug [6].

The slug frequency is determined by the number of slugs that pass through an area of interest during a controlled time interval. The average slug frequency is defined as

$$
\left\langle v_{s}\right\rangle=\frac{1}{N_{k}} \sum_{k=1}^{N_{k}} \frac{1}{\Delta t_{k}},
$$

where the frequency is averaged over the total number of slugs, $N_{k}$.

\subsection{Proper orthogonal decomposition}

Proper orthogonal decomposition produces an organized basis of modes and eigenvalues representing the amount of variance corresponding to each mode. The structures of the flow are organized based on the kernel of the decomposition. Classical POD was introduced to fluid mechanics to analyze turbulent velocity signals [23]. The method of snapshots was modified by Sirovich [26] and applied when the flow measurements contain high spatial resolution in comparison to temporal resolution (cf. Holmes [14]).

For snapshot POD, a spatial correlation matrix is used to compute eigenfunctions, decorrelating structures contained in the snapshots. The two-point spatial correlation tensor is defined as 


$$
\mathbf{R}\left(\mathbf{x}, \mathbf{x}^{\prime}\right)=\frac{1}{N} \sum_{n=1}^{N} \phi\left(\mathbf{x}, t^{n}\right) \phi^{T}\left(\mathbf{x}^{\prime}, t^{n}\right)
$$

where $\phi\left(\mathbf{x}, t^{n}\right)$ is the phase fraction field, $t^{n}$ is the time at a sample $n, N$ refers to the number of snapshots, bold symbols represent vector arrays and the prime represents the spatial coordinate of another point in the domain. $\mathbf{R}\left(\mathbf{x}, \mathbf{x}^{\prime}\right)$ becomes the kernel of the POD. The kernel varies amongst the cases. For the stratified and mixed flow analysis, the kernel is the fluctuating phase fraction. For dispersed and slug flow analysis, the kernel is the instantaneous phase fraction. Assuming the basis modes can be written in terms of the original data and a coefficient $A$, then the basis modes,

$$
\Psi^{i}(\mathbf{x})=\sum_{i=1}^{N} A\left(t^{i}\right) \phi\left(\mathbf{x}, t^{i}\right)
$$

has the largest projection on the phase fraction field in a mean square sense. The solution of the POD integral equation,

$$
\int_{\Omega} \mathbf{R}\left(\mathbf{x}, \mathbf{x}^{\prime}\right) \Psi^{i}\left(\mathbf{x}^{\prime}\right) \mathrm{d} \mathbf{x}^{\prime}=\lambda^{i} \Psi^{i}(\mathbf{x})
$$

yields a complete set of orthogonal eigenfunctions. The substitution of Equations 2.3 and 2.4 into the POD integral equation and the discretization results in an eigenvalue problem described as

$$
\mathbf{C A}=\lambda \mathbf{A}
$$

where the coefficient vector, $\mathbf{A}=\left[A\left(t^{1}\right), A\left(t^{2}\right), \ldots, A\left(t^{n}\right)\right]^{T}, \mathbf{C}$ is a symmetric $N \times N$ matrix with components $C_{j k}=1 / N\left(\phi^{T}\left(\mathbf{x}, t^{j}\right) \phi\left(\mathbf{x}, t^{k}\right)\right)$ and $j, k=1, \ldots, N . \lambda$ is a diagonal matrix 
of $N$ eigenvalues where the eigenvalues and associated eigenmodes are ordered by their proportion of variance, $\lambda^{1}>\lambda^{2}>\lambda^{3}>\cdots>\lambda^{n}$.

According to the eigenvalue problem, the set of coefficients are obtained from the solution of Equation 2.6. The modes are normalized and formed into an orthonormal basis that is defined as

$$
\boldsymbol{\Psi}^{i}(\mathbf{x})=\frac{\sum_{i=1}^{N} A^{i}\left(t^{n}\right) \phi\left(\mathbf{x}, t^{n}\right)}{\left\|\sum_{i=1}^{N} A^{i}\left(t^{n}\right) \phi\left(\mathbf{x}, t^{n}\right)\right\|} .
$$

Using the eigenfunctions of the POD, the phase fraction tomograms may be reconstructed as

$$
\phi\left(\mathbf{x}, t^{n}\right)=\sum_{i=1}^{N} a_{i} \Psi^{i}(\mathbf{x}),
$$

where the set of coefficients, $a_{i}$, are obtained by back-projecting the phase fraction tomograms onto the deterministic POD modes. In the domain $\Omega$, the coefficients are defined as

$$
a_{i}=\int_{\Omega} \phi\left(\mathbf{x}, t^{n}\right) \Psi^{i}(\mathbf{x}) \mathrm{d} \mathbf{x}^{\prime} .
$$

and represent the most prominent features of the flow as a function of time.

A set of eigenfunctions that represent the modes of turbulence and eigenvalues that measure the variance associated with each eigenfunction are provided from the POD analysis. The self-normalized eigenvalues,

$$
K_{n}=\lambda_{n} / \sum_{i=1}^{N} \lambda_{i}
$$


describes the percent of information associated with a given eigenmode $n$. The sum of the eigenvalues shows the distribution of variance, therefore the variance contained in the first $n$ eigenmodes is equal to

$$
Z_{n}=\frac{\sum_{k=1}^{n} \lambda_{k}}{\sum_{i=1}^{N} \lambda_{i}}, \quad n=1,2, \ldots, N
$$




\section{Chapter 3}

\section{Experimental Setup}

\subsection{Well Flow Loop}

Experiments were conducted in the Well Flow Loop of the Institute for Energy Technology in Kjeller, Norway. The test section is constructed of transparent PVC, with a total length of $50 \mathrm{~m}$ and an inner diameter of $100 \mathrm{~mm}$. A schematic of the test section is shown in Figure 3.1 where $F C V$ refers to flow control valves, G1-G4 are the gamma densometers and $d P_{1}-d P_{7}$ are differential pressure transducers.

The gamma densometers measure a single holdup value of the entire cross-section of the pipe while the X-ray CT system measures profiles corresponding to holdup values at different locations along the pipe for more detailed analysis. The pressure and holdup measurements taken from the gamma densometers are averaged over a sampling time of $15 \mathrm{~s}$. Enhanced mixing of the phases is produced by a static mixer oper-

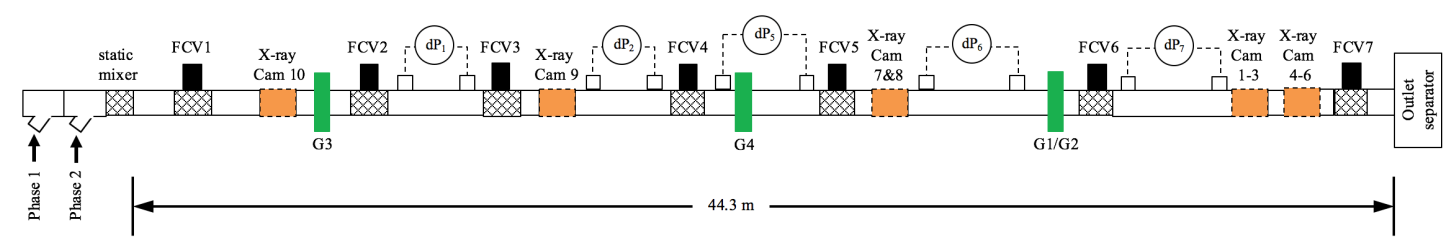

Figure 3.1: Experimental test section (not to scale). 


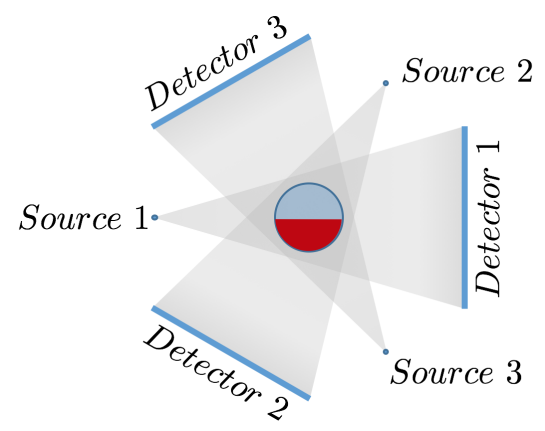

Figure 3.2: Orientation of source and detection devices for one X-ray system along the pipe.

ating directly after the injection site of the two fluids into the pipe. The test section is horizontally aligned at $0 \pm 0.1^{\circ}$.

\subsection{X-ray computed tomography system}

The X-ray tomography system is comprised of up to six point sources and six detectors. The system consists of two triangular setups, each containing three cameras and three sources at adjustable position along the pipe shown by X-ray cameras 1-3 and 4-6 in Figure 3.1. For reference, one triangular setup is shown in Figure 3.2.

Each X-ray is attenuated as it passes through the air, the pipe wall and the fluids inside the pipe, with stronger attenuation indicated by lower intensity of the camera pixel. Calibration images are obtained by filling the pipe entirely with gas, oil or water. This allows for the pixel value to be converted to a phase content percentage value, referred to as holdup value, for the calibrated phases. Two-dimensional tomograms may be constructed from the profiles of holdup measured by X-ray detectors shown in Figure 3.2. The dimension of a single pixel is $0.1 \mathrm{~mm} \times 0.1 \mathrm{~mm}$. For noise reduction, groups of neighboring pixels are averaged and the final resolution is therefore 2 
Table 3.1: Experimental test conditions.

\begin{tabular}{l|ccccc} 
Flow regime & Case & Phase-Phase & $U_{m i x}(\mathrm{~m} / \mathrm{s})$ & X-ray system & Total snapshots \\
\hline Stratified & $05_{\nu} 10_{w c}$ & oil-water & 0.5 & 2 sources & 30 \\
Stratified & $05_{v} 30_{w c}$ & oil-water & 0.5 & 2 sources & 30 \\
Stratified & $05_{\nu} 80_{w c}$ & oil-water & 0.5 & 2 sources & 30 \\
Mixed & $1_{\nu} 10_{w c}$ & oil-water & 1 & 2 sources & 100 \\
Mixed & $1_{v} 30_{w c}$ & oil-water & 1 & 2 sources & 100 \\
Mixed & $1_{\nu} 80_{w c}$ & oil-water & 1 & 2 sources & 100 \\
\hline Dispersed & - & oil-water & 1 & 6 sources & 1232 \\
Slug & - & gas-oil & 1.5 & 6 sources & 1250 \\
\hline
\end{tabular}

mm/pixel.

Table 3.1 shows details of the test parameters. Eight experiments of two-phase flow are analyzed to compare characteristics of the four considered regimes. The stratified and mixed flow cases utilize water and oil as the two-phases. Three cases of both stratified and mixed flow with varying water content of $10 \%, 30 \%$ and $80 \%$ are investigated to better understand dynamics of the flow fields. The velocity and water content values are signified by the subscripts $v$ and $w c$ of the case for future annotation (Table 3.1). Finally, the dispersed flow and slug flow are analyzed separately. The dispersed flow, comprised of oil and water, and slug flow, comprised of gas and oil, utilize X-ray detectors 1-3 and 4-6 for a more detailed construction of the tomogram. Dispersed and slug flow also contain an increased number of snapshots for more detailed analysis. The sum of the velocities of the two phases is the mixed velocity, $U_{m i x}$, obtained from the inlet pump velocity of each fluid. 


\section{Chapter 4}

\section{Results: Stratified and Mixed Flow}

The stratified and mixed flow cases are examined to describe the dynamics of the flow as a function of water content and velocity. The multiple cases considered for each flow regime allow for in-depth analysis of the dominant characteristics, such as interactions between the two fluids, stratification layer geometries and droplet dispersion and accumulation. Trends found via POD will give insight into the dynamical features and phase interactions as a function of the velocity and water content.

\subsection{Time-averaged statistics}

Tomograms of the mean phase fraction of water, $\left\langle\phi_{w}\right\rangle$, for all six cases are shown in Figure 4.1. The spatial coordinates are normalized by the pipe diameter, $D$ and $\langle\cdot\rangle$ denotes ensemble averaging. The three lower velocity cases, in the upper half of Figure 4.1, are in the stratified flow regime, with coherent layers of percent water in the vertical direction. For the $W C=10 \%$ case, the majority of the pipe has uniform phase fraction of pure oil for the upper $7 / 8$ of the pipe. As the water content increases for the low velocity cases, the layers become thicker and more pronounced. For the $W C=30 \%$ case, the stratification initiates near the center of the pipe, continuing to the bottom. The highest water content case shows stratified layers starting near the top of the pipe, 

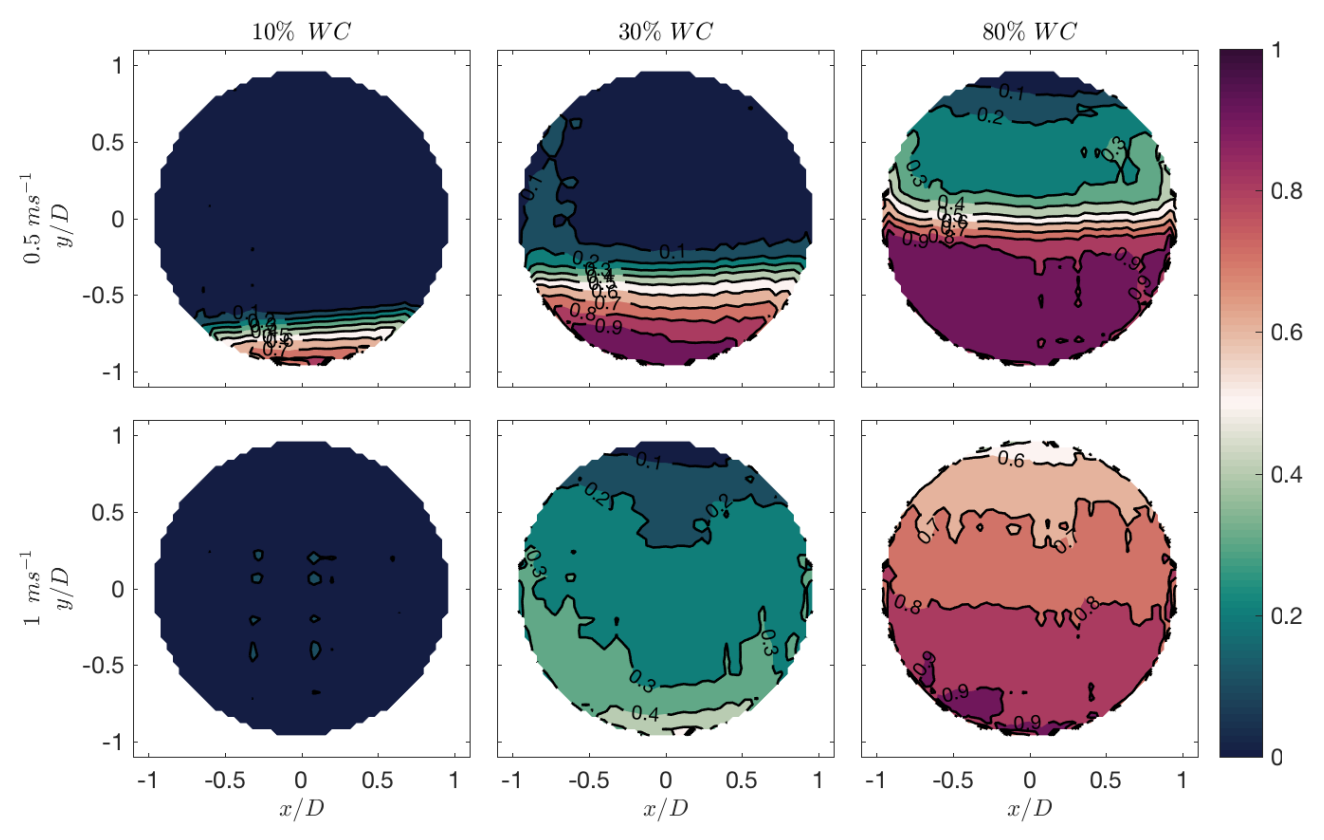

Figure 4.1: Time-averaged tomograms of the cross-sectional water distribution for stratified and mixed flow cases. The time-averaged tomograms show the most prominent characteristics of the flow field. Contour lines indicate the local water fraction in steps of 0.1 .

with $\sim 100 \%$ water phase fraction reached by the center of the pipe. The $1 \mathrm{~m} / \mathrm{s}$ velocity cases indicate a highly mixed flow with the mean phase fraction showing less uniformity as the water fraction changes. Water droplets are more uniformly distributed over the cross-section for the low water content case, $W C=10 \%$. As the water content increases, the dispersed oil starts to increase toward the top of the pipe. For the $W C=$ $30 \%$ and $W C=80 \%$ cases, layers form in the vertical direction, but the majority of the flow does not reach uniform oil or water phase inside the pipe. 


\subsection{Proper orthogonal decomposition}

Figure 4.2 shows the distribution of the important variant structures associated with phase fraction fluctuations as a function of the eigenvalue. In Figure 4.2(A), the amount of variance for a given eigenvalue is shown, where $K_{n}$ is obtained via Equation 2.10. For all six cases the first mode contains the largest portion of variance, with percentages ranging from $5.5 \%$ to $64.4 \%$. The three low velocity cases exhibit a substantial decrease of pertinent information from mode 1 to mode 2 . The highest water content case, $05_{\nu} 80_{w c}$, contains the most information in the first mode. As water content decreases, the value of the first mode also decreases. The large reduction of $K_{n}$ from mode to mode is not seen in the cases with high velocity. This is attributed to the higher level of mixing occurring in the $1 \mathrm{~m} / \mathrm{s}$ velocity cases compared to the stratified flow depicted by the $0.5 \mathrm{~m} / \mathrm{s}$ velocity cases.

The percentage of contribution of the successive eigenvalues is depicted in Figure 4.2(B). The sum of the eigenvalues is equivalent to the information provided by the associated with phase fluctuations, therefore the amount of information contained in the first $n$ eigenvalues is defined by Equation 2.11. In Figure 4.2(B), the three high velocity cases show similar convergence characteristics to each other when compared to the three low velocity cases. This is again due to the amount of mixing occurring when the pipe is running at $1 \mathrm{~m} / \mathrm{s}$. The percent water content also affects the distribution of important structures. At high water content the eigenvalues converge more rapidly though the difference in converges is much less at $v=1 \mathrm{~m} / \mathrm{s}$ than at $v=0.5 \mathrm{~m} / \mathrm{s}$.

Table 4.1 details the corresponding eigenmodes required for reconstructions of the features for $50 \%, 75 \%$ and $95 \%$ of the phase fraction field. The convergence shown in Figure 4.2 is quantified in the table, with trends verified. As is also shown in Fig- 


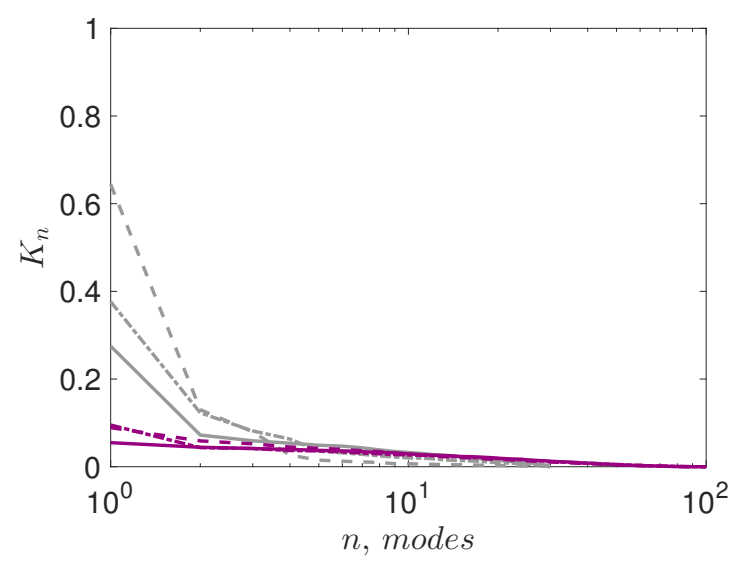

(A)

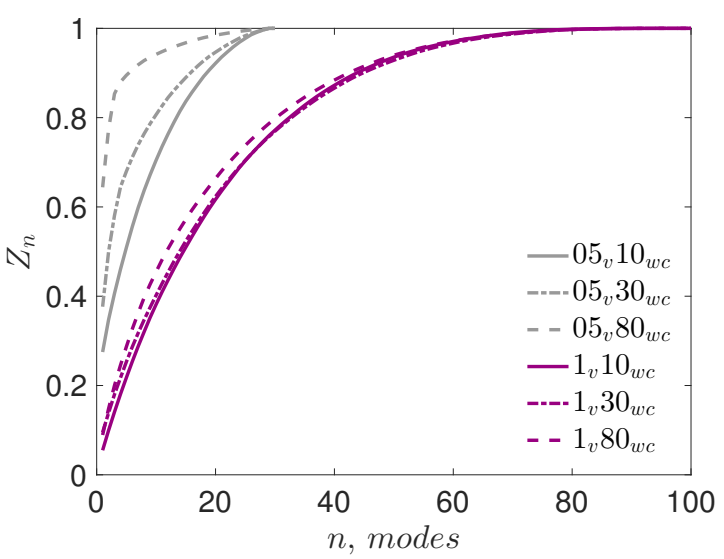

(B)

Figure 4.2: The distribution of variance described by (A) normalized eigenvalues, $K_{n}$, and (B) successive summation of eigenvalues, $Z_{n}$ as a function of the mode, $n$. The value associated with each eigenvalues is representative of the amount of information present in the corresponding eigenmode.

Table 4.1: Corresponding eigenmodes required for 50\%, 75\% and 95\% reconstruction for the stratified and mixed flow cases.

\begin{tabular}{l|cccc} 
Case & $50 \%$ & $75 \%$ & $95 \%$ & Total snapshots \\
\hline $05_{v} 10_{w c}$ & 5 & 12 & 23 & 30 \\
$05_{\nu} 30_{w c}$ & 3 & 8 & 21 & 30 \\
$05_{\nu} 80_{w c}$ & 1 & 2 & 12 & 30 \\
$1_{\nu} 10_{w c}$ & 15 & 29 & 55 & 100 \\
$1_{\nu} 30_{w c}$ & 14 & 29 & 55 & 100 \\
$1_{\nu} 80_{w c}$ & 12 & 26 & 53 & 100 \\
\hline
\end{tabular}

ure 4.2 , the $0.5 \mathrm{~m} / \mathrm{s}$ velocity cases converge more quickly than the high velocity cases. More specifically $50 \%$ reconstruction of the flow is obtained three to twelve fold faster. The convergence is also dependent on water content with increases in water content showing a decreases in modes needed for reconstruction. This trend is more apparent in the slower velocity cases, the high level of mixing involved in the $1 \mathrm{~m} / \mathrm{s}$ velocity cases de-emphasizes the correlation. 
POD modes 1, 2, 3 and 10 are shown for all cases in Figure 4.3. The location and coherence of the modal structures are of interest therefore units of the scale are not included. The first mode of the three lower velocity cases show structures that correspond to the location of stratification of the flow as seen in Figure 4.1. For the higher velocity cases, mode 1 does not contain well defined structures. This correlates to the mean phase fraction fields of the mixed flows depicted in Figure 4.1. As the eigenmodes increase, the features become incoherent. The coherence of the structures present in the modes corresponds to the water content of the cases. The highest water content cases, $05_{\nu} 80_{w c}$ and $1_{\nu} 80_{w c}$ show the highest organization when compared to their respective velocity cases. The structures become less defined as the water content decreases and the two cases with the lowest percent water display incoherence almost immediately.

The convergence of the cases, seen in Figure 4.2 is directly related to the structures present in the eigenmodes. The cases that contain more coherence of the structures, converge quickly when compared with their respective velocity cases. The phase fraction can be described by the eigenmodes more easily due to the larger amount of important structures present in the initial modes and therefore the case converges more quickly. Furthermore, the higher velocity cases show minimal coherence for all eigenmodes due to mixing. This is depicted in Figure 4.2(A) by the gradually decreasing slope of the line for the successive modes.

Using the eigenfunctions of the POD, the phase fraction fluctuations may be reconstructed, and as a result, the interaction between the two phases, $\left\langle\phi_{w}^{\prime} \phi_{o}^{\prime}\right\rangle$ is described as 

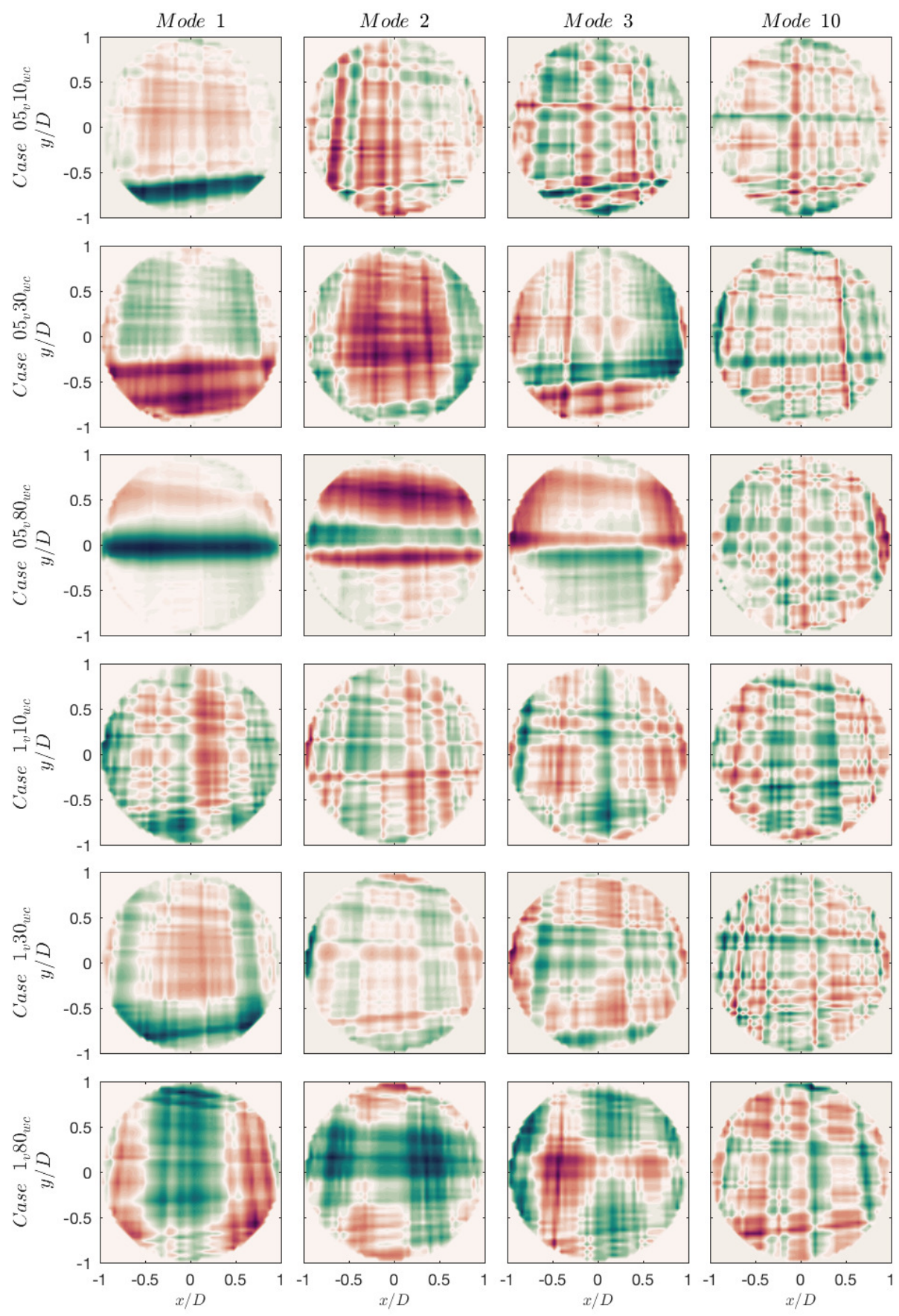

Figure 4.3: Eigenmodes 1, 2, 3 and 10 for all cases. The modes show the most dominant structures of the flow with respect to spatial location. The most important structures are present in the first mode. 


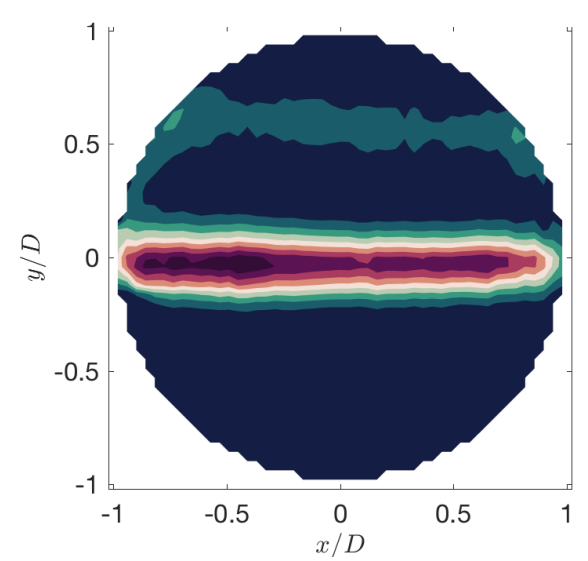

(A)

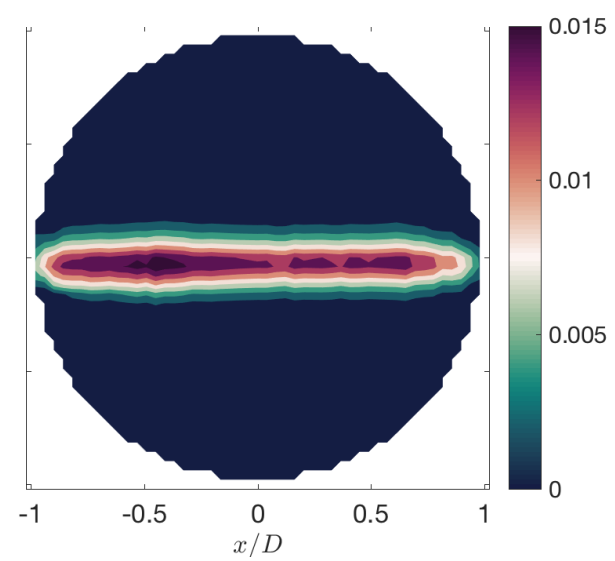

(B)

Figure 4.4: Phase fraction interactions, $\left\langle\phi_{w}^{\prime} \phi_{o}^{\prime}\right\rangle$, of $05_{\nu} 80_{w c}$ for full order description (left) and the 50\% reconstructed reduced order description (right). The low order description shows the main features of the interaction between the two fluids. The reconstruction uses a single mode while the full order description requires all 30 degrees of freedom of the system.

$$
\left\langle\phi_{w}^{\prime}(\mathbf{x}) \phi_{o}^{\prime}(\mathbf{x})\right\rangle=\left\langle\sum_{n=1}^{N} a_{n} \Psi_{w}^{n}(\mathbf{x}) \Psi_{o}^{n}(\mathbf{x})\right\rangle .
$$

Figure 4.4 shows the full order description and a reduced order description of the phase fraction interactions for $05_{\nu} 80_{w c}$ on the left and right, respectively. The reconstructed flow field has been created using one mode, while the full order flow field utilizes all thirty modes. The magnitude of the phase fraction interactions is reduced slightly with the low order reconstruction, but the location and intensity of the interactions are consistent between the two figures. The low order description captures the main features of the phase fraction field. 


\section{Chapter 5}

\section{Results: Dispersed and Slug Flow}

Dispersed flow contains complex flow dynamics due to the droplets of each fluid being enveloped in the other fluid, creating an ill-defined interface between the two-phases. Slug flow, another complex flow regime, is seen when instabilities grow at the interface between the two-fluids due to small perturbations in the flow. Slug flow may lead to safety risks due to the intermittent loading on the pipe walls. Proper orthogonal decomposition of dispersed and slug flow provides mathematical relationships that pertain to the flow dynamics of the respective regime. These relationships may be used to lessen effects of the irregular loading by controlling the flow upstream through condition monitoring.

\subsection{Time-averaged statistics}

Time-averaged tomograms of the mean phase fraction, $\left\langle\phi_{w}\right\rangle$, for the water-oil dispersed flow case and $\left\langle\phi_{o}\right\rangle$, for the gas-oil slug flow case are shown in Figure 5.1. The spatial coordinates are normalized by the pipe diameter, $D$. The mean phase fraction of water for the dispersed flow shows a stratified layer, with a thickness of $0.25 \mathrm{D}$, located near the horizontal centerline of the pipe. There exist two symmetric pockets of $\left\langle\phi_{w}\right\rangle \sim 100 \%$ in the bottom half of the pipe along the outside edges. An area of strat- 


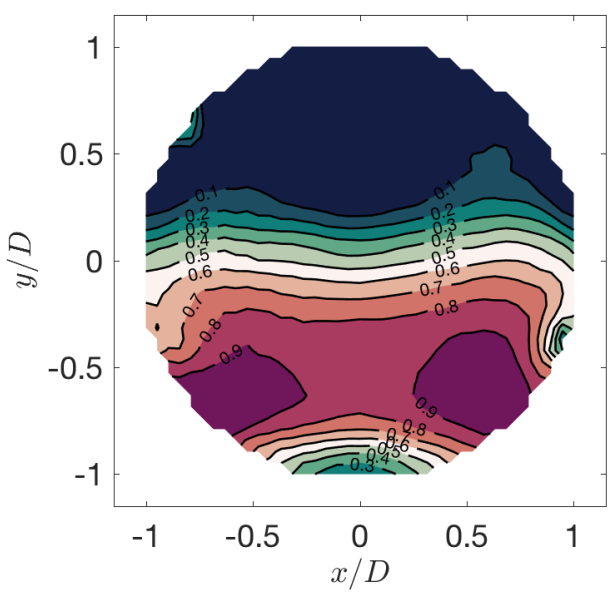

(A)

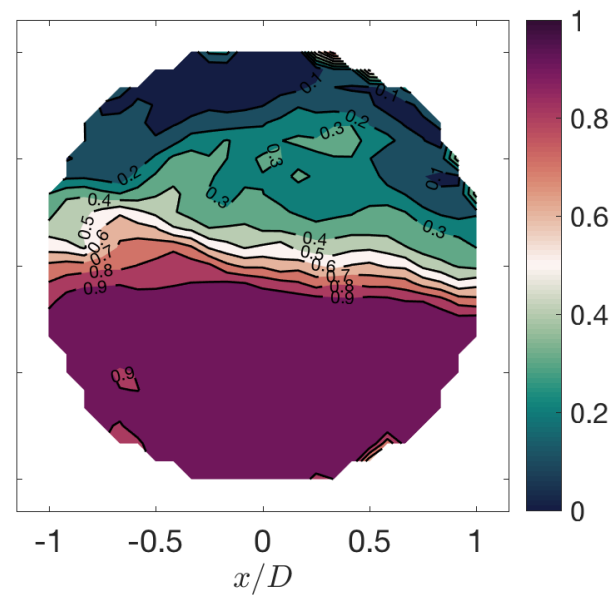

(B)

Figure 5.1: Mean phase fraction tomograms of the cross-sectional distribution (A) $\left\langle\phi_{w}\right\rangle$ for dispersed flow and (B) $\left\langle\phi_{o}\right\rangle$ for slug flow. Most prominent features of the phase fraction field are depicted. Contour lines indicate the local water fraction in steps of 0.1 .

ification is shown along the bottom edge where the phase transforms to majority oil from $y / D=-0.75$ to -1 . Figure 5.1 (B) represents the mean phase fraction of oil over the development and break up of 12 slugs. The mean phase fraction of a single slug has almost identical characteristics with a maximum and mean percent difference of $12 \%$ and $1.7 \%$, respectively. Figure 5.1(B) shows irregular and asymmetric stratification occurring around the horizontal centerline of the pipe. In the bottom half of the pipe, the phase fraction is almost uniformly $100 \%$ oil while the top half is a mixed flow comprised of $\left\langle\phi_{o}\right\rangle \sim 0-0.3$.

Mean slug length of the flow is computed by the experimental holdup detected by the vertically oriented camera with a resulting magnitude of $L_{S} / D=0.24$ and $L / D=$ 10.0. The mean slug frequency, $\left\langle v_{s}\right\rangle$ is $0.0145(1 / \mathrm{s})$. 


\subsection{Proper orthogonal decomposition}

Figure 5.2 depicts the distribution of the variance associated with the instantaneous phase fraction for the the two cases as a function of the eigenvalue. The amount of variance of the eigenvalues is shown in Figure 5.2(A), where again $K_{n}$ is found by Equation 2.10. Both cases show similar trends with the first mode containing the largest portion of variance with $K_{n}=99.5 \%$ and $94.0 \%$ for dispersed and slug flow, respectively. The first mode for each case shows the most dominant structures of the flow field, the mean phase fraction. The first mode of dispersed flow has a greater amount of flow structure details due to the regularity of the instantaneous phase fraction field with respect to time.

Figure 5.2(B) shows the percentage of contribution of the successive eigenvalues as described by Equation 2.11. The same trends are seen as in Figure 5.2(A) with the dispersed flow case converges more quickly than the slug flow with over $99 \%$ of the phase fraction variance present in mode 1 . A convergence of $99 \%$ is reached at mode 3 for the slug flow. This again is due to the uniformity of the flow field field with respect to time.

Eigenmodes 1, 2, 3, 5 and 10 obtained via POD are shown for the dispersed flow field in Figure 5.3. The first mode Figure 5.3(A) shows the mean phase fraction of the flow thus containing significant relative coherence. Mode 2 shows features that correspond to the location of high stratification of the flow seen in Figure 5.1. As the mode increases, Figures 5.3(C)-(E), structures correlate to areas of fluctuating phase fraction and are much less coherent than the first two modes. Mode 100 displays complete incoherence of the phase fraction field.

POD eigenmodes 1-3, 5 and 10 are shown for slug flow in Figure 5.4. Similar to 


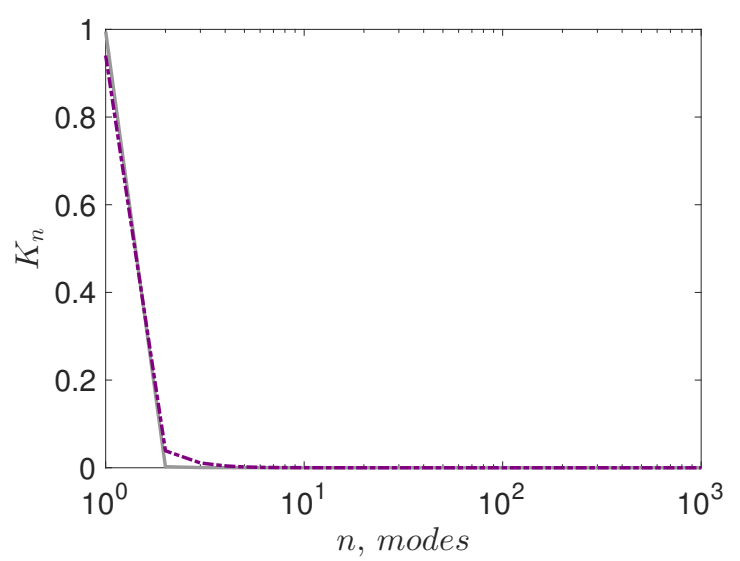

(A)

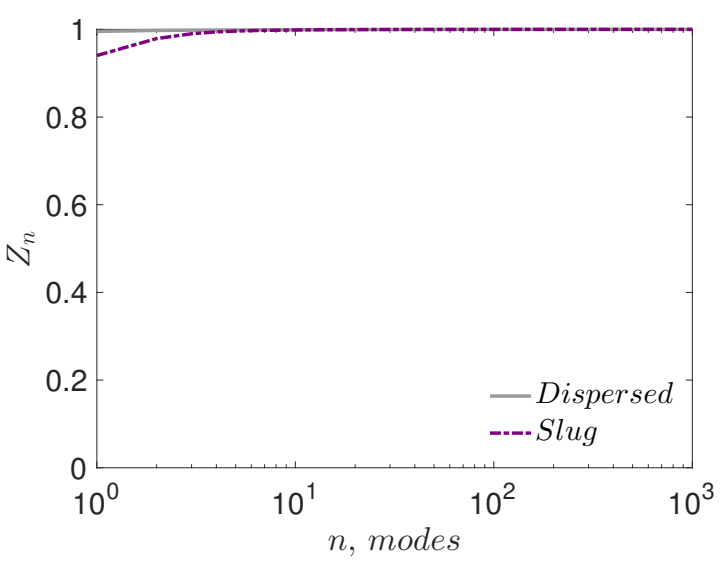

(B)

Figure 5.2: The distribution of variance described by (A) normalized eigenvalues, $K_{n}$, and (B) successive summation of eigenvalues, $Z_{n}$ as a function of the mode, $n$. The value associated with each eigenvalues is representative of the amount of important structures present in the corresponding eigenmode.

the dispersed case the mean phase fraction is characterized by mode 1. Eigenmodes 2, 3 and 5 show structures that correspond to the cyclical development and break up of the slug. The gas bubble develops slowly, indicated by the bias of the structures toward the horizontal centerline of the pipe. More specifically, the oil fills the entire pipe quickly, with oil levels moving from near centerline to the top of the pipe over one or two snapshots. The oil then gradually lowers in the pipe as the top half of the pipe fills with gas, this process takes anywhere from 0.8 to 2.6 seconds. Mode 10 contains minimal coherent structures, following the trend seen by modes 2, 3 and 5 . Figure 5.4(F) shows complete incoherence of the flow field.

The two cases contain different features of the eigenmodes related to the different characteristics of the two flows. The dispersed flow is more uniform, therefore after the initial mode describing the mean phase fraction, only one modes identifies the in- 


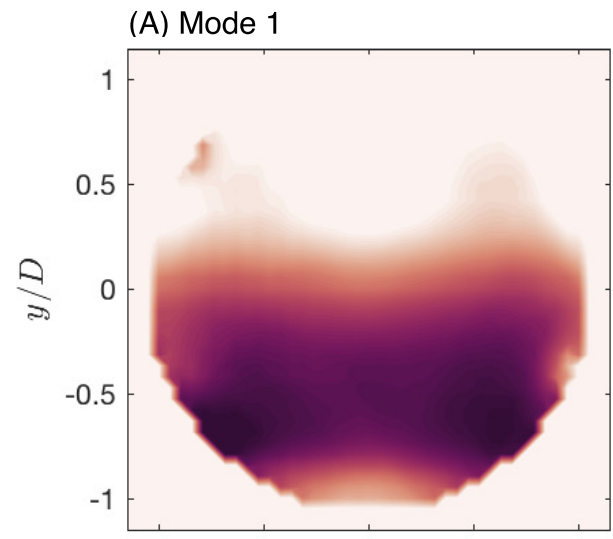

(C) Mode 3

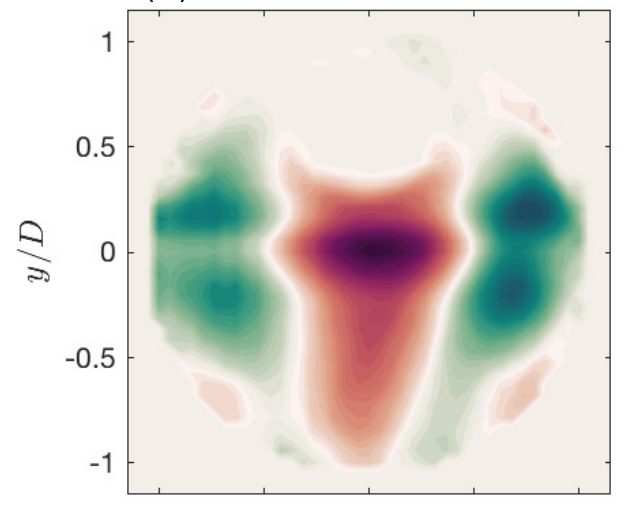

(E) Mode 10

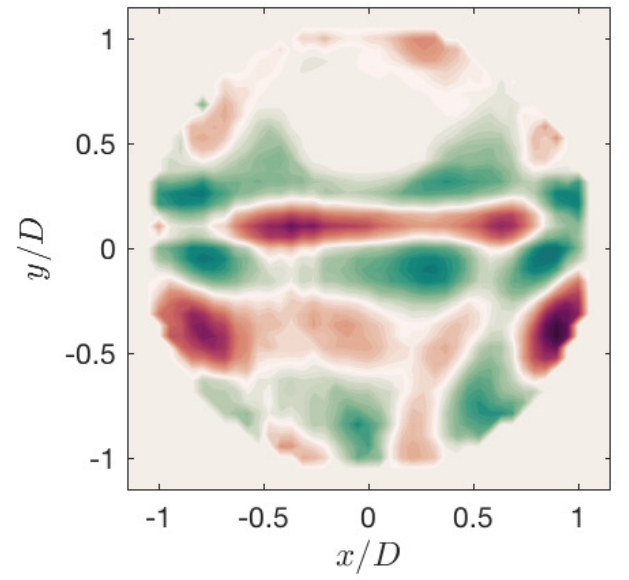

(B) Mode 2

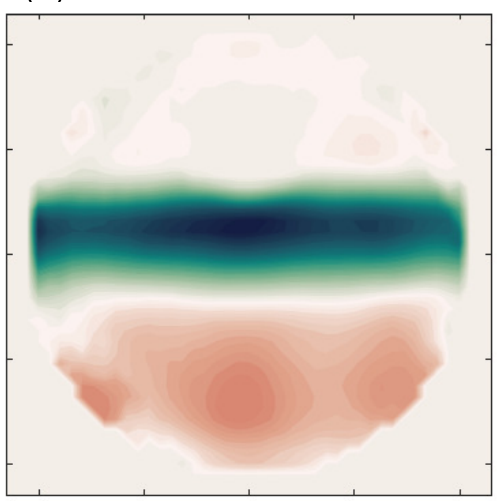

(D) Mode 5

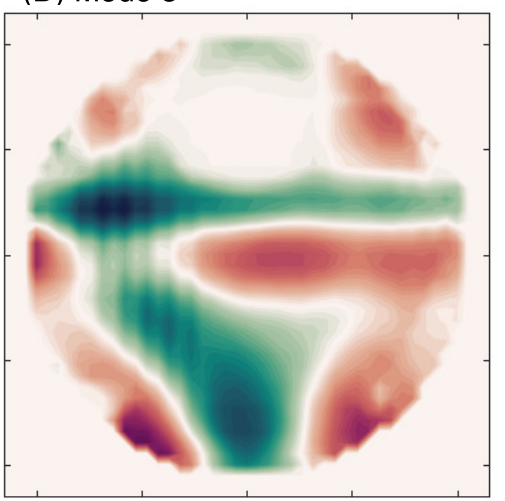

(F) Mode 100

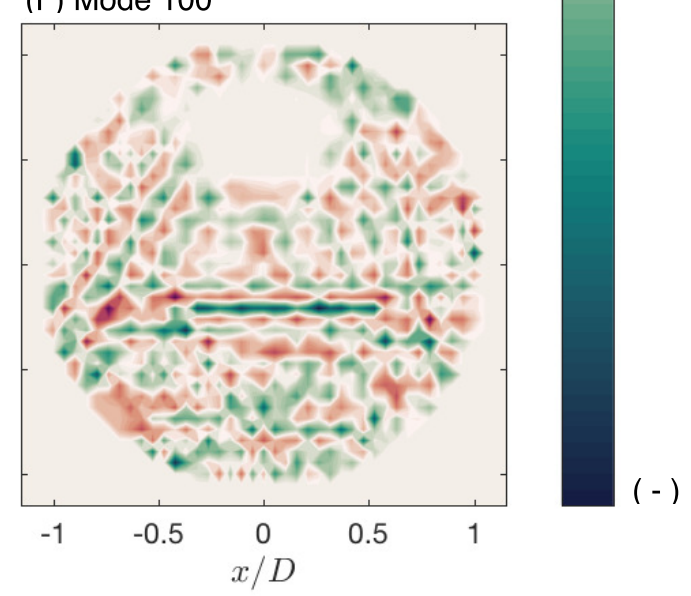

Figure 5.3: Eigenmodes 1, 2, 3, 5, 10 and 100 for the dispersed case depicted in (A) through $(\mathrm{F})$, respectively. The modes show the most dominant structures of the flow in the cross-section of the pipe. The kernel of the POD is the instantaneous phase fraction therefore the most prominent structure is the mean phase fraction, shown in the first mode. 

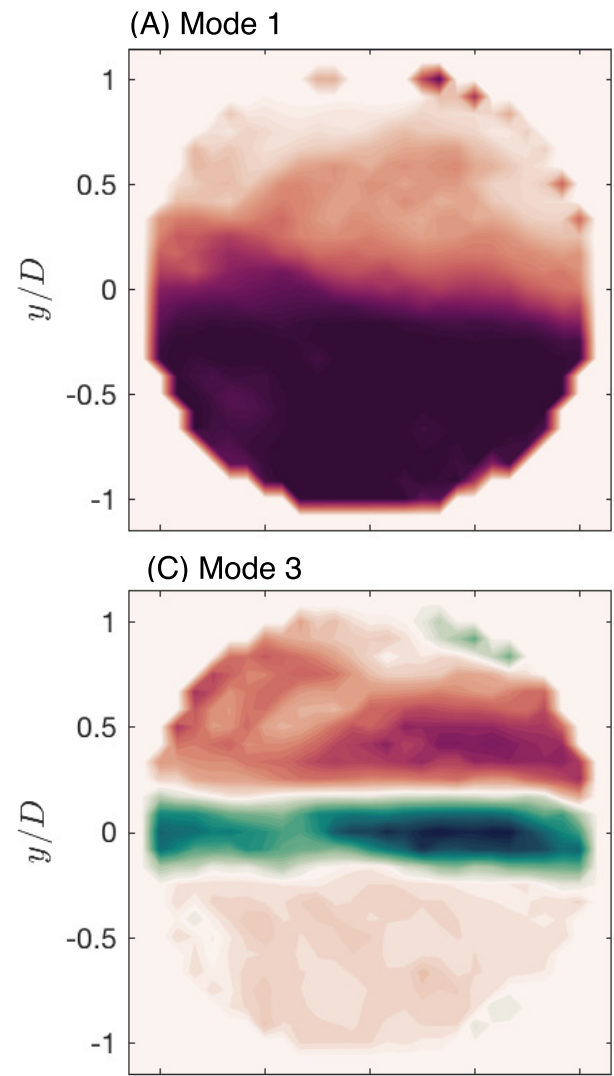

(E) Mode 10

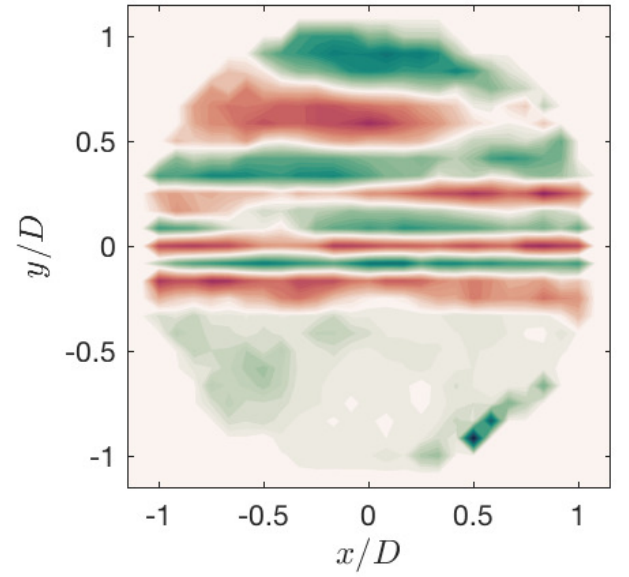

(B) Mode 2

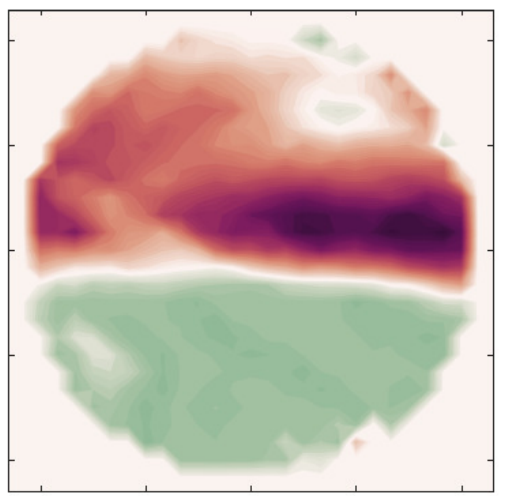

(D) Mode 5

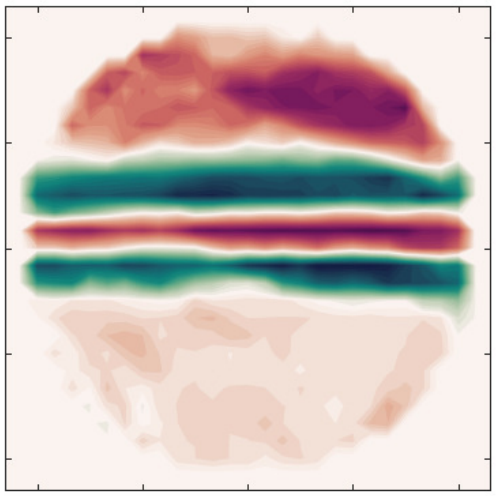

(F) Mode 100

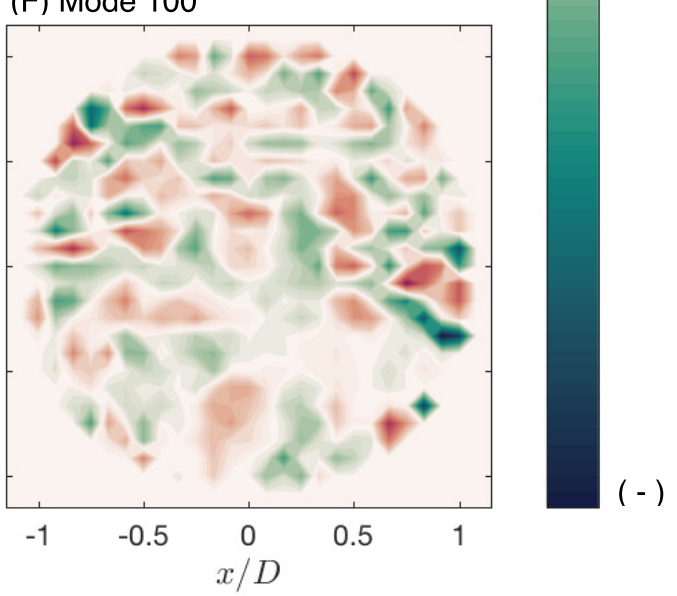

Figure 5.4: Eigenmodes 1, 2, 3, 5, 10 and 100 for the slug flow case depicted in (A) through (F), respectively. The first mode shows the mean phase fraction of the slug flow with successive modes depicting the main features of the flow spatially. 
terface between the fluids. The successive modes, Figures 5.3(C)-(E), show structures that contain negligible significance based on their eigenmode value, attributed to regular variance that occurs during the experiment. The slug flow eigenmodes present more information about the flow field. The structures for modes 2-10 correspond to the slug formation, indicating where the variation is occurring the most in the vertical direction.

Using the temporal coefficients and the eigenfunctions obtained via POD, the instantaneous phase fraction tomograms are reconstructed as seen in Equation 2.8. The full order and reduced order descriptions of the instantaneous phase fraction tomograms of dispersed flow are shown in Figures 5.5(A) and (B), respectively. One mode was used to create the low order description, while the full order flow field utilizes all 1232 modes. Minimal differences are observed between the two figures indicating that dominant characteristics of the flow field are obtained with substantially less degrees of freedom.

In Figure 5.6, the full and reduced order dynamical descriptions of slug flow are shown in (A) and (B), respectively. Here, the reconstructed flow field has been created using three modes, representing $99 \%$ of the energy associated with the phase fraction. The details of the phase fraction tomograms are minimally reduced in the low order reconstructed snapshots, but the location and overall characteristics of the slug are consistent amongst the two figures. The low order description effectively captures the interface between the phases as well as the formation and break up of the slug.

Figure 5.7 shows phase correlation diagrams for POD modes 1, 2 and 3 for dispersed flow and slug flow. The dispersed flow shows minimal correlation between modes 1 and 2 in Figure 5.7(A) and a negligible relationship beyond the first two modes. The 


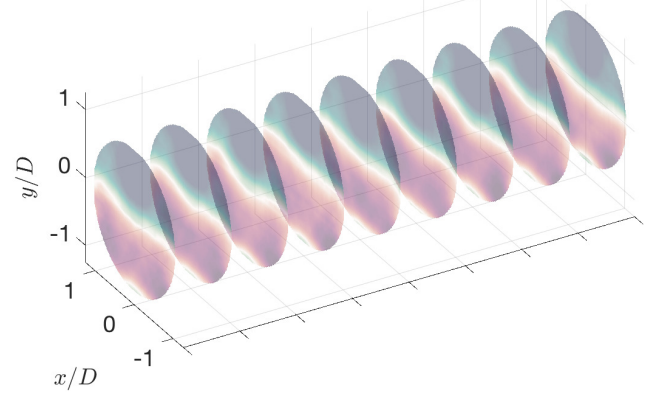

(A)

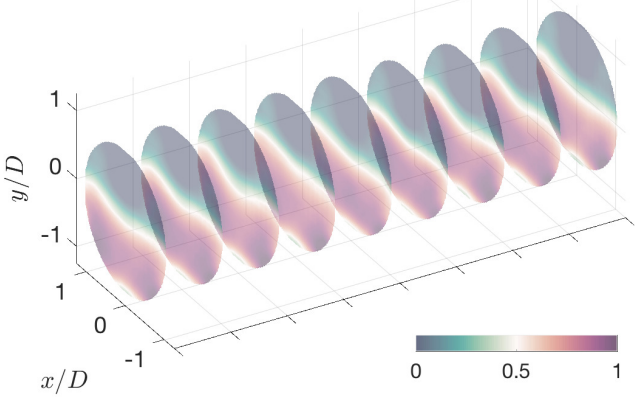

(B)

Figure 5.5: (A) Full order instantaneous phase fraction tomograms and (B) reduced order instantaneous phase fraction tomograms of dispersed flow, reconstructed with 1 eigenmode. The low order description shows main features of the flow while using less than $1 \%$ of the information from the full order model.

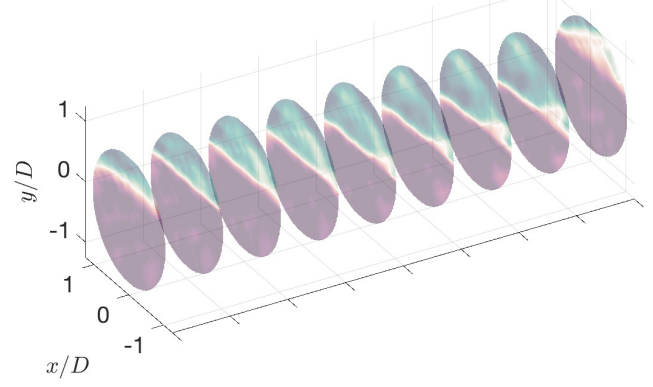

(A)

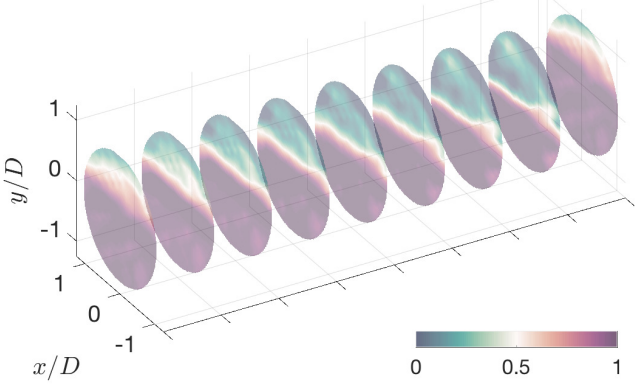

(B)

Figure 5.6: (A) Full order instantaneous phase fraction tomograms and (B) reduced order instantaneous phase fraction tomograms of slug flow, reconstructed with 3 eigenmodes. The reduced order dynamical description includes prominent flow features using three degrees of freedom. The full order description of the flow uses 1250 degrees of freedom. 
linear relationship seen between the modes 1 and 2 is inversely proportional signifying that as $a_{1}$ increases $a_{2}$ decreases. The cyclical characteristics of the slug flow create correlations between the first three modes as seen in Figures 5.7(B), 5.7(D) and 5.7(F). The development of the slug spans the curves as specified in the figures denoted by the asterisk, indicating the start of the slug and the square, signifying the end of the slug. The relationship between $a_{1}$ and $a_{2}$ as well as $a_{2}$ and $a_{3}$ can be described by a 2nd order polynomial, $0.46 x^{2}-12.7 x+84.8$ and $0.17 x^{2}-0.45 x-1.9$, with $R^{2}=0.99$ and 0.93, respectively. The curve depicted by $a_{1}$ and $a_{3}$ is best described by a 3rd order polynomial, $0.24 x^{3}-10.9 x^{2}+165.1 x-827.1$, with $R^{2}=0.92$.

Figure 5.8 shows the two holdup profiles and coefficients $a_{i}$ as a function of time where $i=1,2,3$ and 4 . The included profiles, experimental and centerline holdup, refer to the holdup profile across the pipe detected by the vertically aligned camera and the holdup profile taken as a function of the vertical array located at the center of the pipe, respectively. $t=15-25$ is omitted for clarity, the characteristics of the time steps shown are indicative of the omitted data. In Figure 5.8(A), the experimental holdup time series displays similar trends to the CL holdup, with fluctuations slightly emphasized. This discrepancy between the holdup profiles occurs due to the experimental holdup measurement taking consideration the entirety of the domain. More specifically, experimental holdup includes all $x$ locations and centerline holdup considers only one location at $x / D=0$. The experimental holdup is commonly used in X-ray CT data analysis $[15,25]$ to describe the liquid holdup as a function of vertical position. This quantity directly relates to slug length based on the interface location as a function of time, Equation 2.1. The first coefficient, although larger in magnitude, shows the same trends displayed by the two holdup profiles, denoted as Exp. and CL. 


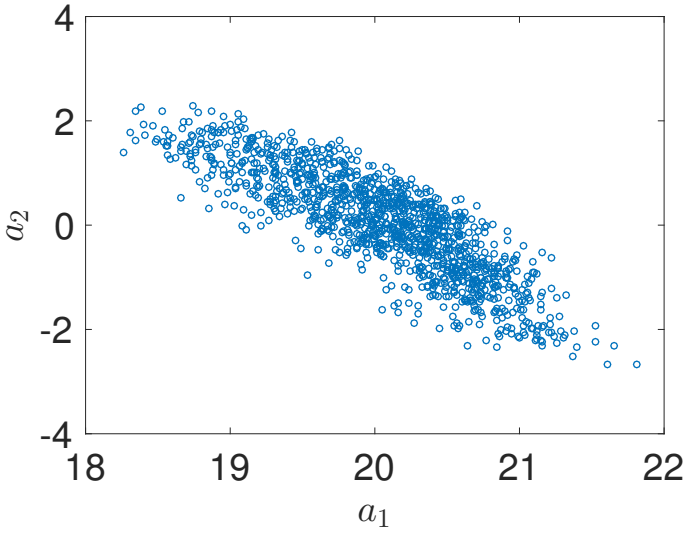

(A)

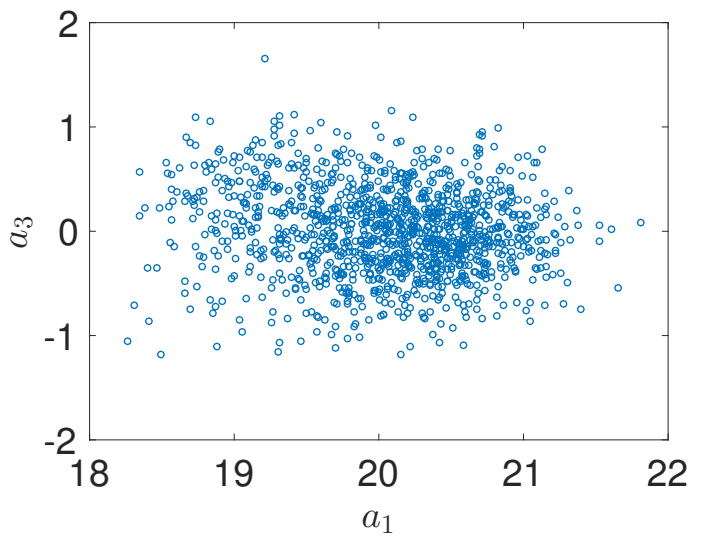

(C)

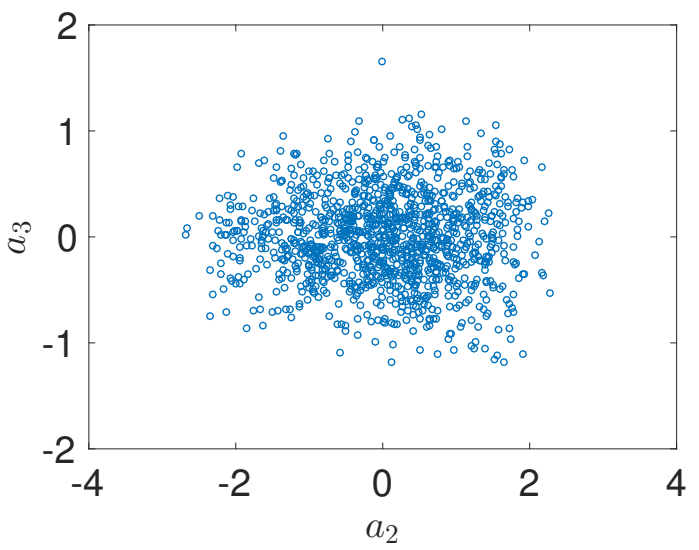

(E)

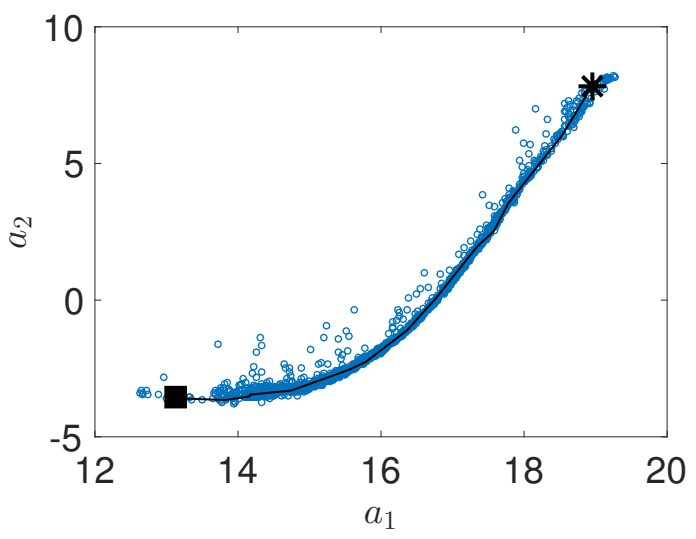

(B)

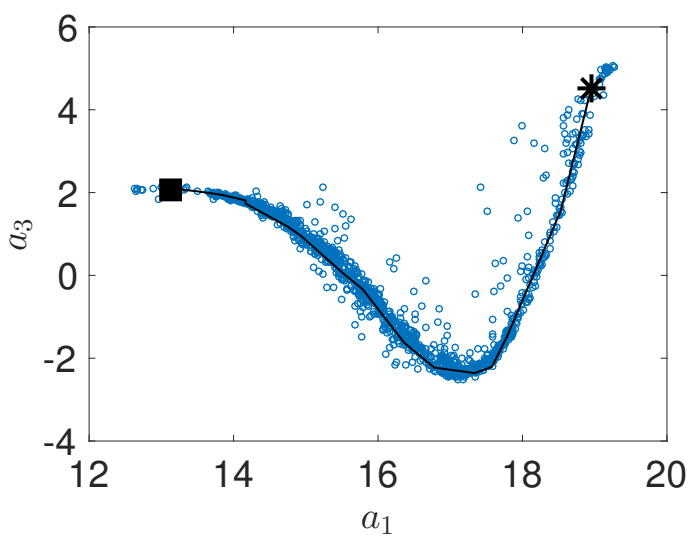

(D)

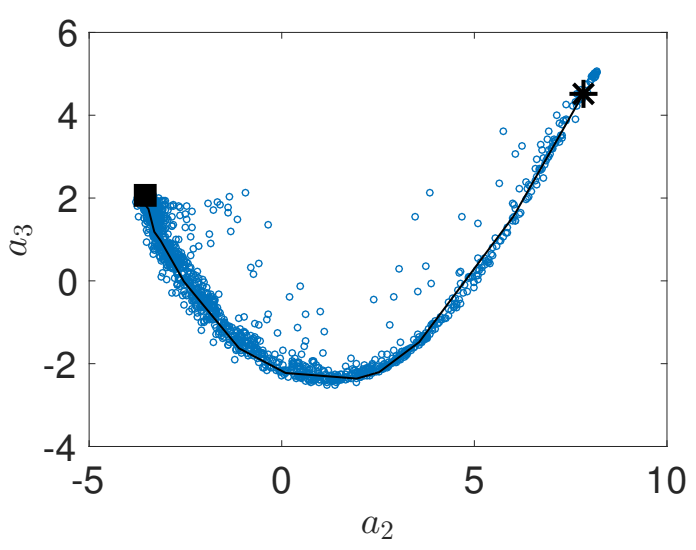

(F)

Figure 5.7: Phase correlation diagrams of temporal coefficients for $i=1,2 \& 3$ for dispersed flow (left column) and slug flow (right column). The asterisk and the square, included in the figures of slug flow, represent the formation at $t=t_{o}$ and break up at $t=t_{f}$ of the slug, respectively. 
$a_{2}$ shows an inverse relation to $a_{1}$ as well as the experimental and CL holdup profiles. As the temporal coefficient increases, $a_{3}$, the trends are muted compared to the first two coefficients, with $a_{4}$ showing negligible fluctuations. The dispersed flow shows fluctuations occurring within a small bandwidth. The variance of the holdup around $y / D=0$ is minimal when compared with mean phase fraction, depicted by $a_{1}$. The variance around $y / D=0$ seen here is directly related to the energetic structure shown in Figure 5.3(B).

The holdup profiles in Figure 5.8(B) exhibit cyclical fluctuations as the slug develops and breaks down. Similar to the dispersed case, the experimental holdup curve shows an emphasized version of the CL holdup curve. The first coefficient displays the same tendencies revealed by the holdup profiles but with dulled features. As the coefficient increases, $a_{2}$, the CL holdup is described well by the coefficient with the mean percent difference between the two curves at $8.7 \%$. As the coefficient increases, $i=3$, the coefficient shows a similar tendency to phase holdup during the first few time instances surrounding the slug formation. Directly after the slug development, $a_{3}$ exhibits inverse fluctuations to those displayed by the two holdup profiles. By $a_{4}$ the fluctuations of the curve are slightly muted with tendencies similar to the inverse properties of the third coefficient.

The descriptions of the slug development by the second coefficient correlate to slug length analysis as well. The mean slug length computed by the second temporal coefficient, $L_{s} / D=10.0$, is the same magnitude as the mean slug length found from the experimental holdup in Section 5.1. Similarly, the mean slug frequency for both methods of analysis $\left\langle v_{s}\right\rangle=0.0145(1 / \mathrm{s})$. The largest variation between the slug length found from experimental holdup and from the POD temporal coefficients is $3.6 \%$ for this data 
set.

The relationship between the first coefficients and the holdup profiles is directly related to the phase correlation diagrams shown in Figure 5.7. The inverse correlation seen in Figure 5.7(A) is validated by the direct relation shown between the fluctuations of $a_{1}$ and the holdup profiles and the inverse correlation between $a_{2}$ and the holdup curves. Also the lack of correlation between the remaining coefficients in Figures 5.7(C) and (E) verify that the third coefficient does not contain prominent characteristics of the flow field.

For the slug flow case, the correlation between $a_{1}$ and $a_{2}$ shown in Figure 5.7(B) is verified. The first two coefficients show similar trends with $a_{2}$ showing more emphasized representations of the holdup when compared to $a_{1}$. The third coefficient shows a direct relationship to the phase holdup profiles during slug development and an inverse relationship as the slug breaks up and that can be seen as it relates to $a_{1}$ and $a_{2}$ in Figures 5.7(D) and (F). The third coefficient shows a direct correlation for the beginning of the slug then after a minimum is reached, is inversely related for the breaking up of the slugs. 


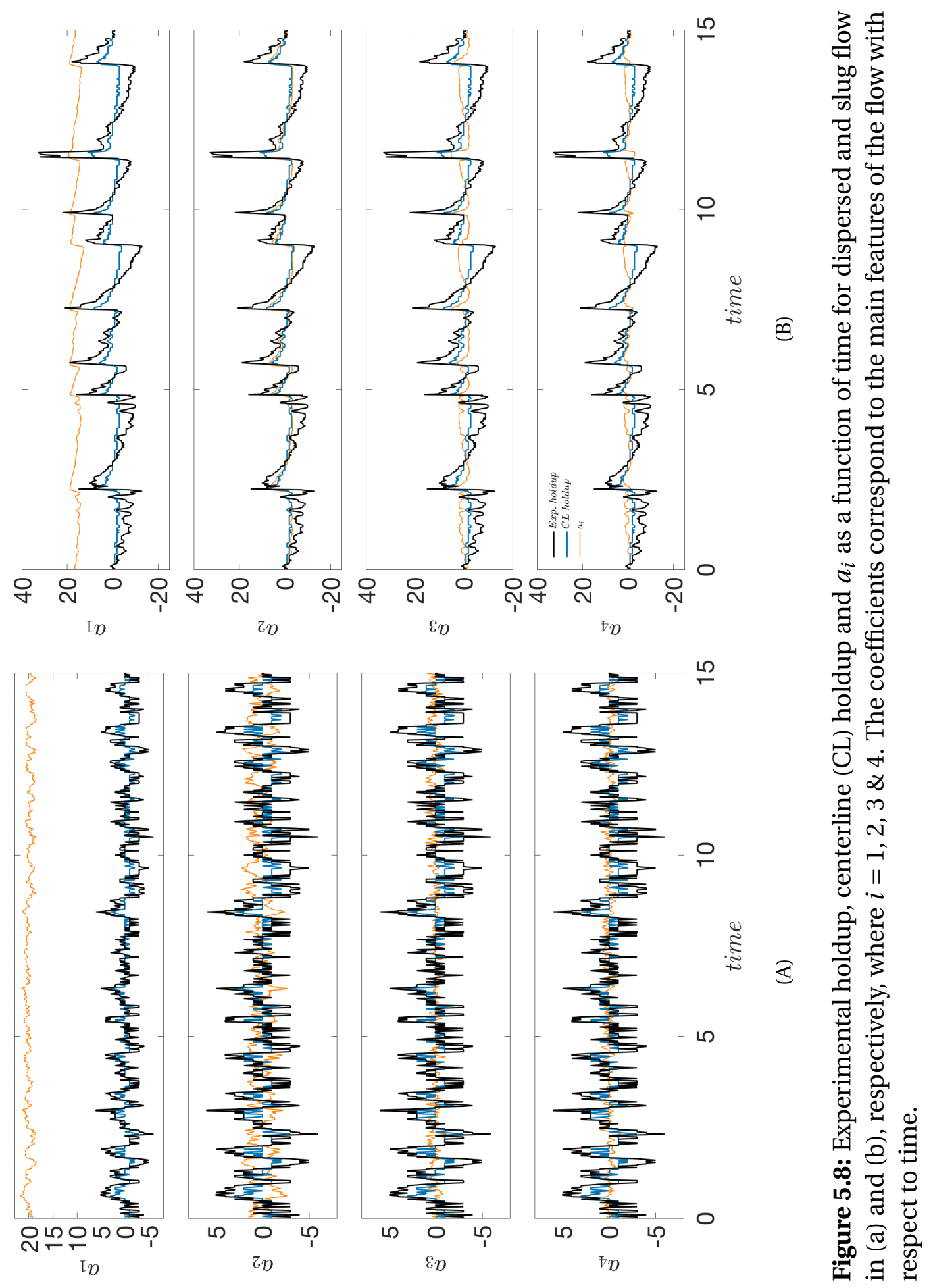




\section{Chapter 6}

\section{Conclusion}

Experiments were investigated to further understand phase fraction characteristics for stratified, mixed, dispersed and slug flow. Proper orthogonal decomposition was performed on all cases as well as statistical analysis on slug flow. For stratified flow, the locations of coherent structures in the first few modes correspond to the locations of the liquid-liquid interface. The same trend is not seen in the mixed flow cases, where $v=1 \mathrm{~m} / \mathrm{s}$. The pronounced interface due to the high mixing of oil and water create nearly incoherent structures in all modes. This observation is validated by the slow convergence of the modes when compared to the low velocity cases. It was also observed that for stratified flow, the main characteristics of the flow are described with a small number of modes.

Mean slug length found via statistical analysis of experimental holdup and from the second temporal coefficient were in perfect agreement. Over the sampling time of $25 \mathrm{~s}$, the dispersed flow is uniform with small fluctuations present at the liquidliquid interface. The liquid holdup of slug flow follows a periodic profile as a function of time as slugs form and break up, with variation in frequency between the liquid slugs. The dispersed case converges to $99 \%$ in the first mode, while slug flow reaches

$99 \%$ convergence by mode 3 . The eigenmodes show coherent features of the flow in 
only the first few modes with mode 1 showing the mean phase fraction of the flow for both cases. The interface of the fluids is depicted in mode 2 for dispersed flow with little coherence seen in successive modes. The interface of the slug flow exists at $0<y / D<1$ and is constantly in motion, which is seen by the layering of structures in modes 2, 3, 5 and 10. Reconstructions, showing dominant features of the two flow fields, are obtained with 1 and 3 modes for dispersed flow and slug flow, respectively. Slug flow shows high dependence between temporal coefficients for $i=1,2$ and 3 . There exists a strong correlation between the dependent coefficients and the liquid holdup. For dispersed flow this correlation is observed for modes 1 and 2. For slug flow, this correlation is seen in the first four coefficients. The second coefficient, $a_{2}$, describes the centerline holdup of the slug flow with high accuracy.

The mathematical framework provided by POD via the eigenfunctions and temporal coefficients describe the dominant features with minimal degrees of freedom of the systems. This translates to a large reduction of computational costs for further analysis of the flow field. The descriptions provided can also be correlated to other flow parameters that are more easily implemented and less expensive than the X-ray CT system. The relationship between the POD framework and the easily measured flow parameters can be used to develop flow control tools, improve multiphase simulations and advance predictive methodologies in numerous industrial applications. 


\section{Chapter 7}

\section{Future Work}

Building on the recognized relationships between holdup and temporal coefficients, examination of correlations between flow characteristics obtained via POD and easily measurable flow parameters, for example instantaneous pressure fluctuations, will be carried out. If relationships exist between the temporal coefficients and other parameters of the flow, then models can be constructed to identify variations in the flow characteristics upstream from the location of interest as well as potential flow control schemes to avoid such regimes. These systems can be implemented to improve condition monitoring in many industrial applications.

Critical points will also be identified via the second derivative test by analyzing the Hessian. Comparisons will be investigated between critical points and the tomograms as well as the eigenmodes. The maxima, minima and saddle points obtained from the second derivative test may be used to identify key locations in the cross-section of the pipe, and further characterize the structures of the POD eigenmodes. The critical points will also be compared with the dynamics of the flow shown by the temporal coefficients. This comparison will relate the liquid holdup development with respect to time and critical points at various spatial locations within the pipe. Poincaré maps will also be produced to further study the dynamical system. 
Finally, a low order dynamical system will be formulated for the flow fields. This dynamical system will generate a set of parameters based on the POD temporal coefficients to describe the flow. The system can then be descreetly calibrated based on simpler flow parameters through transfer functions at specified time intervals to provide short and long term predictions of the flow dynamics. 


\section{Bibliography}

[1] E. Al-SAFran, C. SARICA, H.-Q. Zhang, AND J. Brill, Investigation of slug flow characteristics in the valley of a hilly-terrain pipeline, International journal of multiphase flow, 31 (2005), pp. 337-357.

[2] B. BAJIC, Multidimensional diagnostics of turbine cavitation, American Society of Mechanical Engineers Journal of Fluid Engineering, 124 (2002), pp. 943-950.

[3] S. BAlachandar And J. K. EATON, Turbulent dispersed multiphase flow, Annual Review of Fluid Mechanics, 42 (2010), pp. 111-133.

[4] N. Brauner and A. Ullmann, Modeling of phase inversion phenomenon in twophase pipe flows, International Journal of Multiphase Flow, 28 (2002), pp. 11771204.

[5] T. A. Brenner, R. L. Fontenot, P. G. Cizmas, T. J. O’Brien, and R. W. Breault, A reduced-order model for heat transfer in multiphase flow and practical aspects of the proper orthogonal decomposition, Computers \& Chemical Engineering, 43 (2012), pp. 68-80.

[6] J. N. E. Carneiro, R. Fonseca Jr, A. J. Ortega, R. C. Chucuya, A. O. NieckELE, AND L. F. A. AZEVEDO, Statistical characterization of two-phase slug flow in a horizontal pipe, Journal of the Brazilian Society of Mechanical Sciences and Engineering, 33 (2011), pp. 251-258.

[7] P. G. Cizmas, A. Palacios, T. O’Brien, and M. Syamlal, Proper-orthogonal decomposition of spatio-temporal patterns in fluidized beds, Chemical engineering science, 58 (2003), pp. 4417-4427.

[8] M. COOK AND M. BeHnia, Slug length prediction in near horizontal gas-liquid intermittent flow, Chemical Engineering Science, 55 (2000), pp. 2009-2018.

[9] M. P. Dudukovic, F. LARAChi, And P. L. Mills, Multiphase reactors-revisited, Chemical Engineering Science, 54 (1999), pp. 1975-1995. 
[10] X. Escaler, E. Egusquiza, M. Farhat, F. Avellan, and M. Coussirat, Detection of cavitation in hydraulic turbines, Mechanical systems and signal processing, 20 (2006), pp. 983-1007.

[11] C. Fu, L. Ye, Y. Liu, R. Yu, B. Iung, Y. Cheng, And Y. Zeng, Predictive maintenance in intelligent-control-maintenance-management system for hydroelectric generating unit, IEEE transactions on energy conversion, 19 (2004), pp. 179-186.

[12] G. U. HANYANG AND G. U. O. LIEJIN, Stability of stratified gas-liquid flow in horizontal and near horizontal pipes, Chinese Journal of Chemical Engineering, 15 (2007), pp. 619-625.

[13] V. Hasmatuchi, M. Farhat, S. Roth, F. Botero, and F. Avellan, Experimental evidence of rotating stall in a pump-turbine at off-design conditions in generating mode, Journal of Fluids Engineering, 133 (2011), p. 051104.

[14] P. Holmes, J. L. Lumley, And G. Berkooz, Turbulence, coherent structures, dynamical systems and symmetry, Cambridge University Press, 1998.

[15] B. Hu, M. LAngsholt, L. LiU, P. Andersson, And C. Lawrence, Flow structure and phase distribution in stratified and slug flows measured by $x$-ray tomography, International Journal of Multiphase Flow, 67 (2014), pp. 162-179.

[16] B. Hu, C. Stewart, C. P. Hale, C. J. Lawrence, A. R. W. Hall, H. Zwiens, and G. F. HEwITT, Development of an x-ray computed tomography (ct) system with sparse sources: application to three-phase pipe flow visualization, Experiments in fluids, 39 (2005), pp. 667-678.

[17] R. I. IsSA AND M. H. W. KEMPF, Simulation of slug flow in horizontal and nearly horizontal pipes with the two-fluid model, International journal of multiphase flow, 29 (2003), pp. 69-95.

[18] U. Kertzscher, A. Seeger, K. Affeld, L. Goubergrits, and E. Wellnhofer, $X$-ray based particle tracking velocimetry-a measurement technique for multiphase flows and flows without optical access, Flow Measurement and Instrumentation, 15 (2004), pp. 199-206.

[19] P. Kumar AND R. P. SAInI, Study of cavitation in hydro turbines - A review, Renewable and Sustainable Energy Reviews, 14 (2010), pp. 374-383.

[20] O. Kvernvold, V. Vindøy, T. Søntvedt, A. SaAsen, and S. Selmer-Olsen, Velocity distribution in horizontal slug flow, International journal of multiphase flow, 10 (1984), pp. 441-457. 
[21] R. LindKen AND W. MERZKIRCH, A novel piv technique for measurements in multiphase flows and its application to two-phase bubbly flows, Experiments in fluids, 33 (2002), pp. 814-825.

[22] L. Liu, O. K. Matar, C. J. Lawrence, And G. F. HewitT, Laser-induced fluorescence (lif) studies of liquid-liquid flows. part $i$ : flow structures and phase inversion, Chemical engineering science, 61 (2006), pp. 4007-4021.

[23] J. L. LuMLEY, The structure of inhomogeneous turbulent flows, Atmospheric turbulence and radio wave propagation, (1967), pp. 166-178.

[24] H. SchÜmann, M. TutKun, And O. J. Nydal, Experimental study of dispersed oil-water flow in a horizontal pipe with enhanced inlet mixing, Part 2: In-situ droplet measurements, Journal of Petroleum Science and Engineering, 145 (2016), pp. 753-762.

[25] H. SchÜmann, M. Tutkun, Z. YAng, AND O. J. Nydal, Experimental study of dispersed oil-water flow in a horizontal pipe with enhanced inlet mixing, part 1: Flow patterns, phase distributions and pressure gradients, Journal of Petroleum Science and Engineering, 145 (2016), pp. 742-752.

[26] L. Sirovich, Turbulence and the dynamics of coherent structures. Part I: Coherent structures, Quarterly of applied mathematics, 45 (1987), pp. 561-571.

[27] Y. TAitel AND A. E. DUKLER, A model for predicting flow regime transitions in horizontal and near horizontal gas-liquid flow, AIChE Journal, 22 (1976), pp. 4755.

[28] B. D. WoOds, Z. FAn, AND T. J. HANRATTY, Frequency and development of slugs in a horizontal pipe at large liquid flows, International Journal of Multiphase Flow, 32 (2006), pp. 902-925.

[29] I. ZADRAZIL, O. K. MATAR, AND C. N. MARKIDES, An experimental characterization of downwards gas-liquid annular flow by laser-induced fluorescence: Flow regimes and film statistics, International Journal of Multiphase Flow, 60 (2014), pp. 87-102. 